\title{
OPEN Role of the EF-hand and coiled-coil domains of human Rab44 in localisation and organelle formation
}

\author{
Kohei Ogawa ${ }^{1,2,3}$, Tomoko Kadowaki $^{1 凶}$, Mitsuko Tokuhisa ${ }^{1,2,3}$, Yu Yamaguchi $^{2}$, \\ Masahiro Umeda ${ }^{3} \&$ Takayuki Tsukuba ${ }^{2 \bowtie}$
}

Rab44 is a large Rab GTPase that contains an amino-terminal EF-hand domain, a coiled-coil domain, and a carboxyl-terminal Rab GTPase domain. However, the roles of the EF-hand and coiled-coil domains remain unclear. Here, we constructed various deletion and point mutants of human Rab44. When overexpressed in HeLa cells, the wild-type Rab44 (hWT) formed ring-like structures, and partially localised to lysosomes. The dominant negative mutant, hT847N, localised to lysosomes and the cytosol, while the constitutively active mutant, h0892L, formed ring-like structures, and partially localised to the plasma membrane and nuclei. The $\mathrm{h} \Delta \mathrm{EF}, \mathrm{h} \Delta$ coil, and $\mathrm{h} 826-1021$ mutants also formed ring-like structures; however, their localisation patterns differed from hWT. Analysis of live imaging with LysoTracker revealed that the size of LysoTracker-positive vesicles was altered by all other mutations than the hC1019A and $\mathrm{h} \Delta \mathrm{EF}$. Treatment with ionomycin, $\mathrm{a} \mathrm{Ca}^{2+}$ ionophore, induced the translocation of hWT and $\mathrm{h} \Delta$ coil into the plasma membrane and cytosol, but had no effect on the localisation of the $\mathrm{h} \Delta \mathrm{EF}$ and $\mathrm{h}$ 826-1021 mutants. Thus, the EF- hand domain is likely required for the partial translocation of Rab44 to the plasma membrane and cytosol following transient $\mathrm{Ca}^{2+}$ influx, and the coiled-coil domain appears to be important for localisation and organelle formation.

Intracellular membrane trafficking coordinates complex network systems that precisely regulate various membrane-bound organelles in cells ${ }^{1,2}$. Dynamic intracellular movements such as vesicle transport, membrane fission, tethering, docking, and fusion events are centered on Rab GTPases acting as critical regulators ${ }^{3,4}$. Rab GTPases translocate between the cytosol and membranes by undergoing conformational changes that are strictly controlled by two important regulators: the guanine nucleotide exchange factor (GEF) and GTPase activating protein (GAP) ${ }^{5,6}$. GEFs convert the inactive GDP-bound Rab protein to the active GTP-bound form on membranes, while GAPs catalyse the active GTP-bound Rab protein to the inactive GDP-bound form ${ }^{7,8}$. In addition, lipid modification is also an important characteristic of Rab GTPases. For the membrane binding functions, Rab GTPases contain one or two cysteine residues in the carboxy-terminus (C-terminus) that undergo posttranslational modification through prenylation ${ }^{9,10}$. To date, approximately 70 members of Rab GTPases have been identified in mammals ${ }^{11,12}$.

Among these, Rabs 1-43 are small Rab GTPases with a molecular weight of about $20-30 \mathrm{kDa}^{13}$. In contrast, Rab44, Rab45, and CRACR2A (also known as Rab46) are large Rab GTPases that contain a few additional domains including EF-hand and coiled-coil domains in addition to the Rab-GTPase domain ${ }^{14-16}$. Rab44 was recently identified as a protein that is upregulated during osteoclast differentiation by our research group ${ }^{17}$. Using knockdown (with small interfering RNA) and overexpression systems, we showed that Rab44 negatively regulates osteoclast differentiation by modulating intracellular calcium levels following NFATc1 activation. Comparing the sequence of the human and mouse Rab44 reveals that both Rab44 proteins contain EF-hand, coiled-coil, and Rab-GTPase domains. Initially, mouse Rab44 was identified as only a short form lacking the EF-hand domain ${ }^{17}$. However, our recent studies reported that mouse mast cells and bone-marrow cells have two isoforms: the long form including the EF-hand domain and the short form lacking this domain ${ }^{18,19}$. These findings have

${ }^{1}$ Department of Frontier Oral Science, Graduate School of Biomedical Sciences, Nagasaki University, Sakamoto 1-7-1, Nagasaki 852-8588, Japan. ${ }^{2}$ Department of Dental Pharmacology, Graduate School of Biomedical Sciences, Nagasaki University, Sakamoto 1-7-1, Nagasaki 852-8588, Japan. ${ }^{3}$ Department of Clinical Oral Oncology, Graduate School of Biomedical Sciences, Nagasaki University, Sakamoto 1-7-1, Nagasaki 852-8588, Japan. ${ }^{\square}$ email: tomokok@nagasaki-u.ac.jp; tsuta@nagasaki-u.ac.jp 
a

human Rab44 (WT)

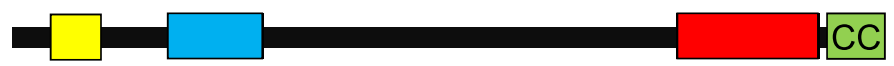

$\mathrm{h} \Delta \mathrm{EF}$

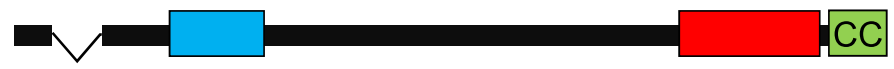

$\mathrm{h} \Delta \mathrm{coil}$

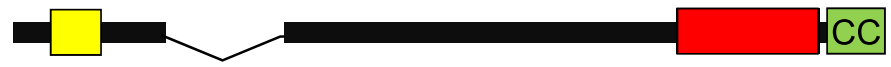

h826-1021

hT847N

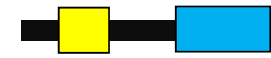

$\mathrm{N} \quad \mathrm{CC}$ DN

hQ892L

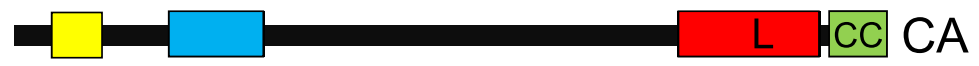

hC1019A

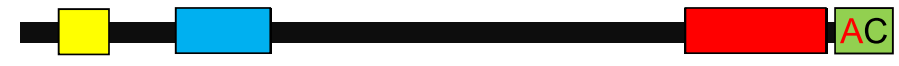

hC1020A

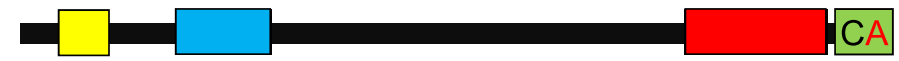

hC1019/1020A

b

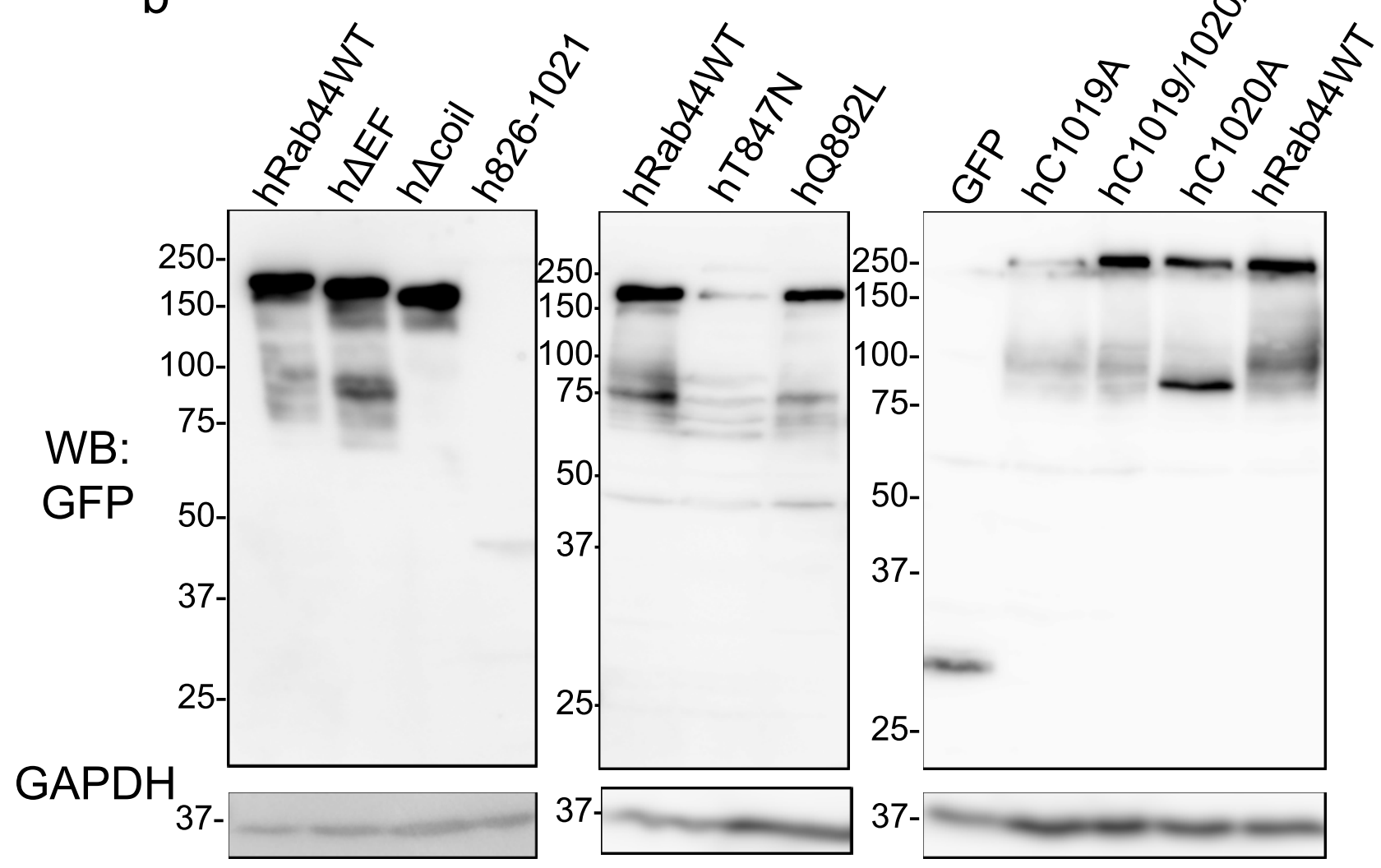

Figure 1. Construction and ectopic expression of wild type and various Rab44 mutants in HeLa cells. (a) Schematic representation of wild type and mutant Rab44 expressed in HeLa cells. The EF-hand, coiled-coil, Rab family, and lipidation domains are shown in yellow, blue, red, and green respectively. The recombinant Rab44 proteins were expressed as N-terminal GFP-fusion proteins. (b) Western blot analysis of HeLa cell expressing Rab44 and its derivatives. 
raised fundamental questions about the differences in localisation and function of Rab44 between humans and mice. Indeed, our recent study using the murine macrophage cell line, RAW-D cells, showed that mouse Rab44 localised mainly to lysosomes and the Golgi complex ${ }^{17}$. There are currently no studies on the localisation of human Rab44. Moreover, the roles of the amino-terminal EF-hand and coiled-coil domains in the localisation of human Rab44 remain unclear.

In this study, we investigated the mechanisms involved in the localisation of human Rab44 by ectopically expressing various Rab44 mutants in HeLa cells, which are human epithelial cells most often used in intracellular localisation analyses.

\section{Results}

Ectopic expression and localisation of wild type and mutants of human Rab44 in HeLa cells. To investigate the subcellular localisation of human Rab44, we constructed various mutants of human Rab44 by site-directed mutagenesis (Fig. 1a), including wild-type human Rab44 (hWT), an EF-hand motif deletion ( $\mathrm{h} \Delta \mathrm{EF}$ ), a coiled-coil domain deletion ( $\mathrm{h} \Delta$ coil), a Rab domain only (h826-1021), a constitutively active (CA) mutant (hQ892L), a dominantly negative (DN) mutant (hT847N), and C-terminal lipidation site mutants with point mutations at residues 1019 and 1020 (C1019A, C1020A, and C1019A/C1020A). The hWT and mutant constructs were exogenously expressed as N-terminal green fluorescent protein (GFP)-fusion proteins in HeLa cells (Fig. 1a). The protein levels of the mutants in the HeLa cells were confirmed by western blot analysis (Fig. 1b). The expressed proteins were detected at their predicted molecular weights, although the expression levels of h826-1021, hT847N, and C1019A were relatively low.

The ectopically expressed hWT formed ring-like structures, and partially surrounded the LAMP1-positive lysosomes in HeLa cells (Fig. 2a). However, the hWT hardly merged with EEA1 (a marker for early endosomes)and GM130 (a marker for the Golgi complex)-positive compartments. (Fig. 2a). Interestingly, the hWT partially merged with KDEL [a marker for endoplasmic reticulum (ER)]-positive compartments. Quantitative analysis of colocalisation between the hWT and these organelle markers is shown in Fig. 2b. We determined that an average score of 0.4 or more is colocalised, 0.2 to 0.4 is partially colocalised, and score of 0.2 or less is not colocalised. Similar determinations were performed in subsequent experiments.

The DN mutant, hT847N, was mostly diffuse, indicating cytosolic localisation, and colocalised partially with LAMP1-positive lysosomes and slightly with the KDEL-positive ER (Fig. 3a). However, hT847N failed to merge with EEA1-positive early endosomes and the GM130-positive Golgi complex (Fig. 3a). Intriguingly, HeLa cells expressing the DN mutant hT847N were rounder in shape than those expressing hWT (see Fig. 2). Quantification of colocalisation between the hT847N and these organelle markers is also shown in Fig. $3 \mathrm{~b}$.

The CA mutant, hQ892L, formed ring-like structures and was partially detectable in LAMP1- and EEA1positive compartments, the plasma membrane, and the nuclei, but hardly detectable in the GM130- and KDELpositive compartments (Fig. 3c). Of note, in hQ892L-expressing HeLa cells, the shape of the cells and the nuclei were distorted, the cell-cell contact sites were unclear, and hQ892L appeared to promote cell-cell adhesion (Fig. 3c). These results were confirmed by quantitative analysis of colocalisation between the hQ892L and these organelle markers (Fig. 3d).

One of the lipidation-site mutants, hC1019A, was diffusely distributed throughout the cytoplasm, although it partially localised to the KDEL-positive compartments (Fig. 3e). However, the hC1019A mutant was hardly detectable in the LAMP1-, GM130-, and EEA1-positive compartments (Fig. 3e). Consistent with these results, hC1019A colocalisation analysis revealed a colocalisation score of 0.4 with KDEL, and only 0.2 or less with other organelle markers (Fig. 3f).

The other lipidation-site mutant, hC1020A, was also diffusely detected in the cytoplasm, and partially colocalised with LAMP1- and KDEL-positive compartments (Fig. 3g). Indeed, quantitative analysis of colocalisation of hC1020A yielded colocalisation values of $0.2-0.4$ with LAMP1 and KDEL (Fig. 3h).

The double mutant hC1019/1020A was also mostly diffusely distributed in the cytoplasm with partial colocalisation with KDEL-positive compartments, and was undetectable in other organelles (Fig. 3i). Quantification of colocalisation between the hC1019/1020A and these organelle markers validated these observations (Fig. 3j).

The $\mathrm{h} \triangle \mathrm{EF}$ mutant formed ring-like structures, which were partially merged with LAMP1-positive lysosomes (Fig. 4a). The h $\triangle$ EF mutant did not colocalise with EEA1-, GM130-, or KDEL-positive compartments (Fig. 4a). Quantitative analysis of colocalisation between the $\mathrm{h} \Delta \mathrm{EF}$ mutant and these organelle markers indicated its localisation pattern differed from hWT. (Fig. 4b).

The $\mathrm{h} \Delta$ coil mutant formed ring-like structures and mainly merged with LAMP1-positive lysosomes, but not with EEA1-, GM130-, or KDEL-positive compartments (Fig. 4c). Quantification of the colocalisation of h $\Delta$ coil mutant with these organelle markers showed that its localisation pattern differed from hWT (Fig. 4d). The colocalisation score of the $\mathrm{h} \Delta$ coil mutant with LAMP1-positive lysosomes was higher than that of $\mathrm{hWT}$, and that with KDEL-positive compartment was lower than that of hWT, suggesting that fusion of the $\mathrm{h} \Delta$ coil mutant with lysosomes is promoted but fusion with the ER is reduced (Fig. $4 \mathrm{~d}$ ).

The h826-1021 mutant mostly formed ring-like structures, although it partially induced vesicle-like structures (Fig. 4e). The h826-1021 mutant colocalised with LAMP1-positive lysosomes, but not with other organelle markers (Fig. 4e), confirmed in quantitative colocalisation analysis (Fig. 4f). Similar to the h $\Delta$ coil mutant, the h826-1021 mutant also had a higher colocalisation coefficient with LAMP1-positive lysosomes and a lower one with KDEL-positive compartment compared with hWT (Fig. 4f).

Effects of human Rab44 and its mutants on formation of LysoTracker-positive compartments in live HeLa cells. To investigate the effects of the hWT and mutant constructs on formation of acidic compartments, we measured the size of LysoTracker-positive vesicles in live Hela cells expressing human Rab44 

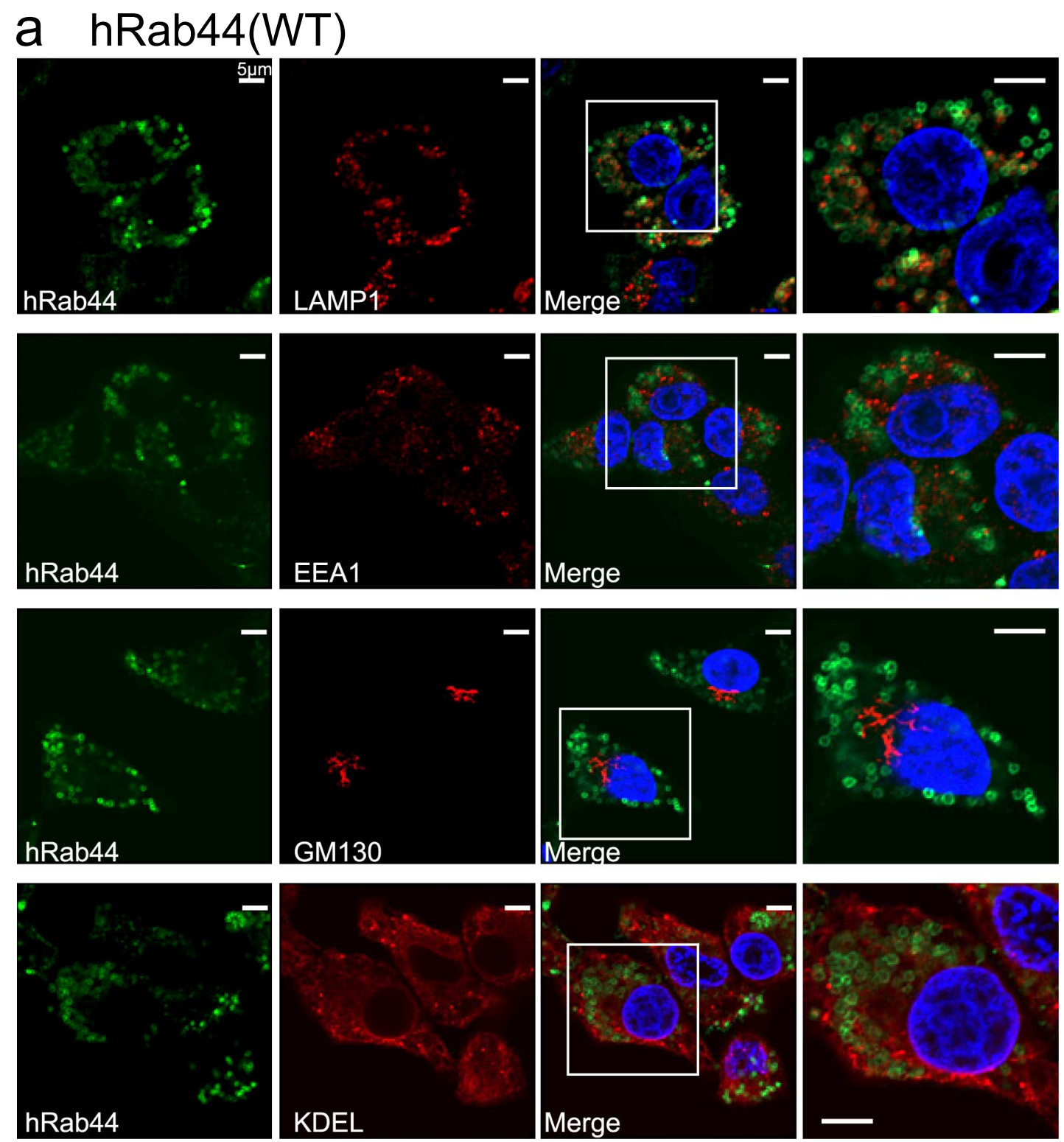

b

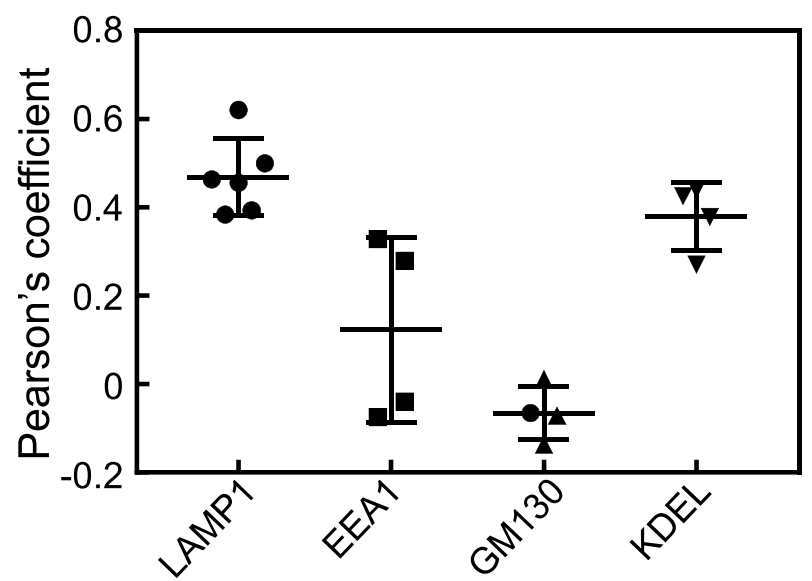

Figure 2. Subcellular localisation of eGFP-hRab44 (hWT) in HeLa cells. (a) Confocal laser microscopic analysis of HeLa cells immunofluorescently stained for LAMP1 (marker for late endosomes/lysosomes), EEA1 (marker for early endosomes), GM130 (marker for the Golgi), and KDEL (marker for ER). Bar: $5 \mu$ m. (b) Quantitative analysis of the ratio of colocalisation of hWT with indicated organelle markers. Data are mean \pm SD $(\mathrm{n} \geq 4)$. 


\section{a $\mathrm{hT} 847 \mathrm{~N}$}
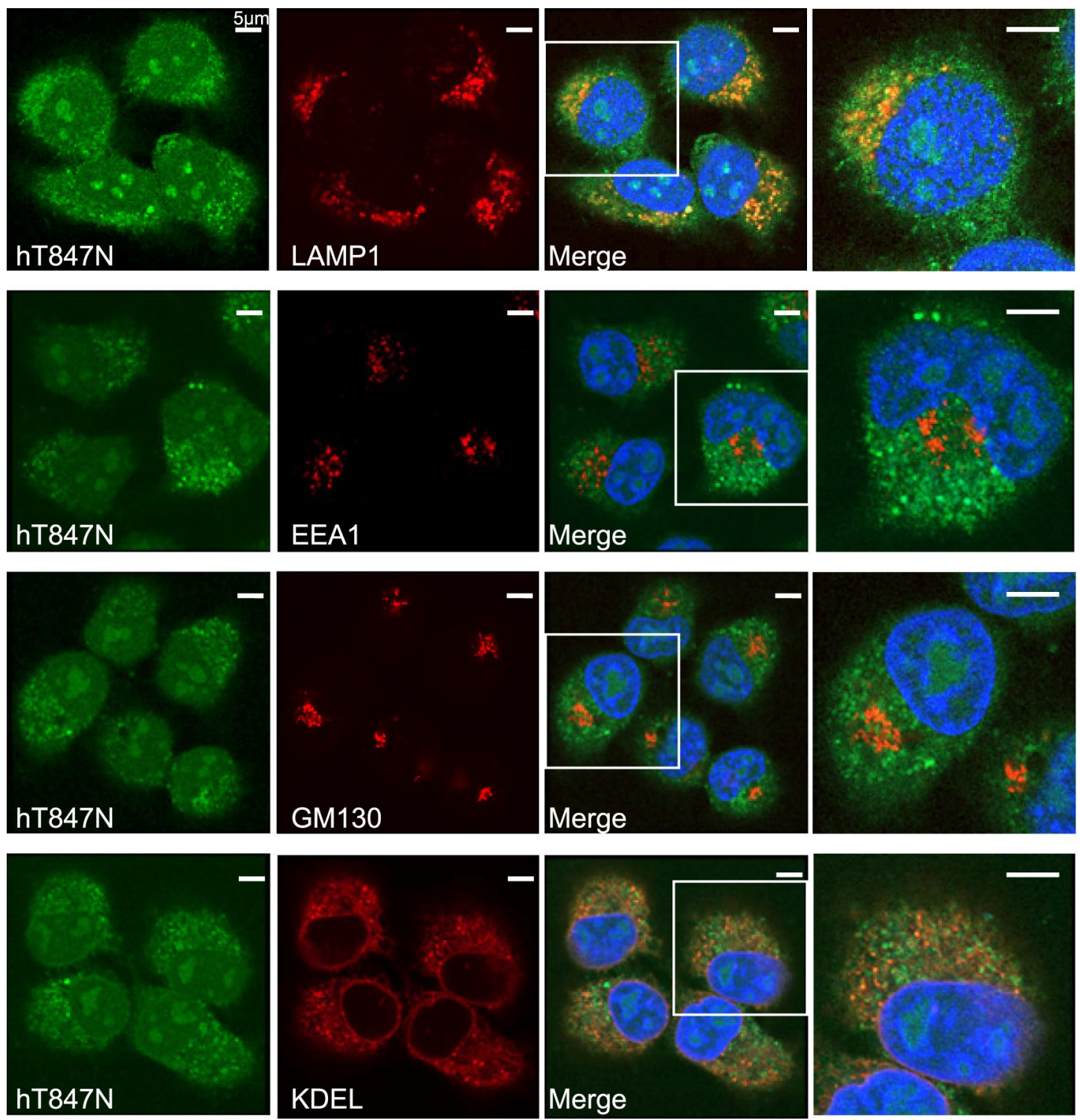

b

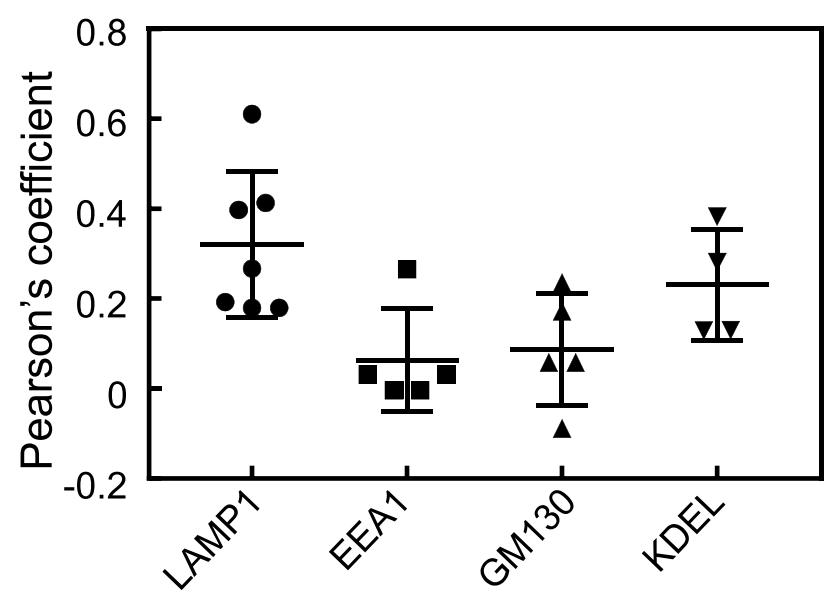

Figure 3. Subcellular localisation of hRab44 mutants in HeLa cells. (a,b) eGFP-hT847N (DN) in HeLa cells, (c,d) eGFPhQ892L (CA), (e,f) eGFP-hC1019A. (g,h) eGFP-hC1020A, and (i,j) eGFP-hC1019/1020A. (a, c, e, g, i) Confocal laser microscopic analysis of HeLa cells immunofluorescently stained for LAMP1 (marker for late endosomes/lysosomes), EEA1 (marker for early endosomes), GM130 (marker for the Golgi), and KDEL (marker for ER). Bar: $5 \mu \mathrm{m} .(\mathbf{b}, \mathbf{d}, \mathbf{f}, \mathbf{h}, \mathbf{j}$ ) Quantitative analysis of the ratio of colocalisation of these mutants with these organelle markers. Data are mean \pm SD ( $\mathrm{n} \geq 4$ ). 


\section{C hQ892L}
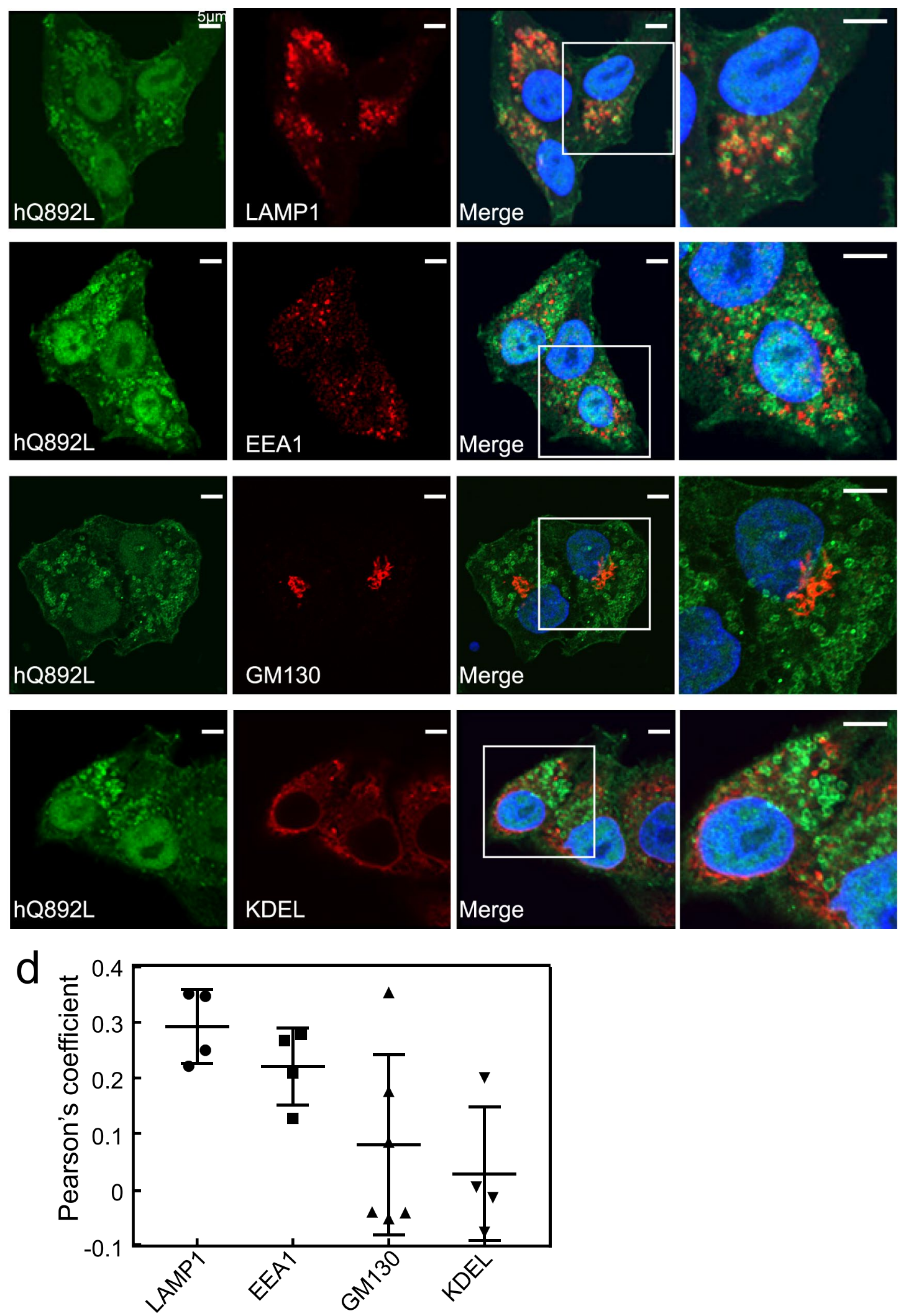

Figure 3. (continued) 


\section{e $\mathrm{hC} 1019 \mathrm{~A}$}
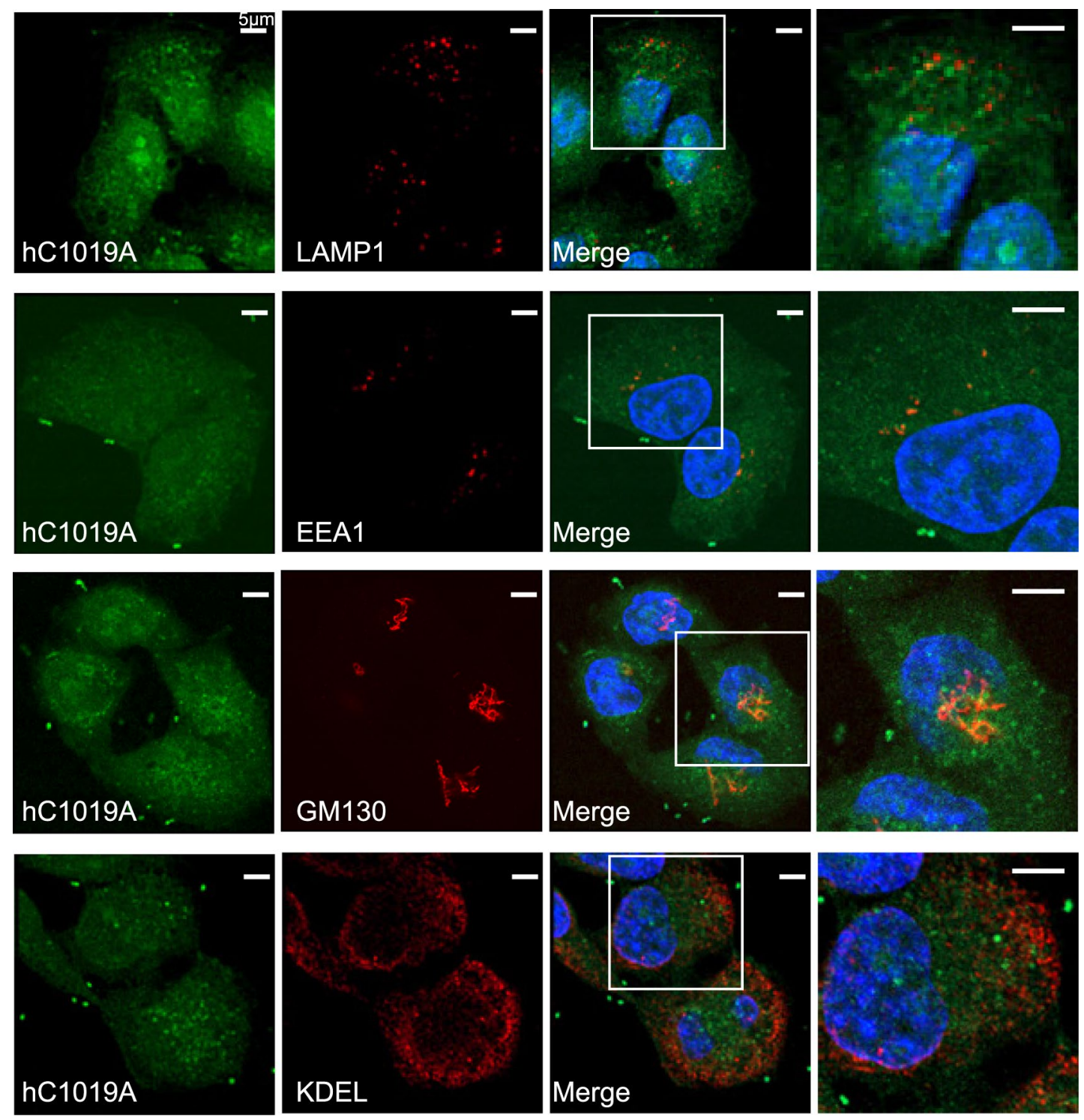

f

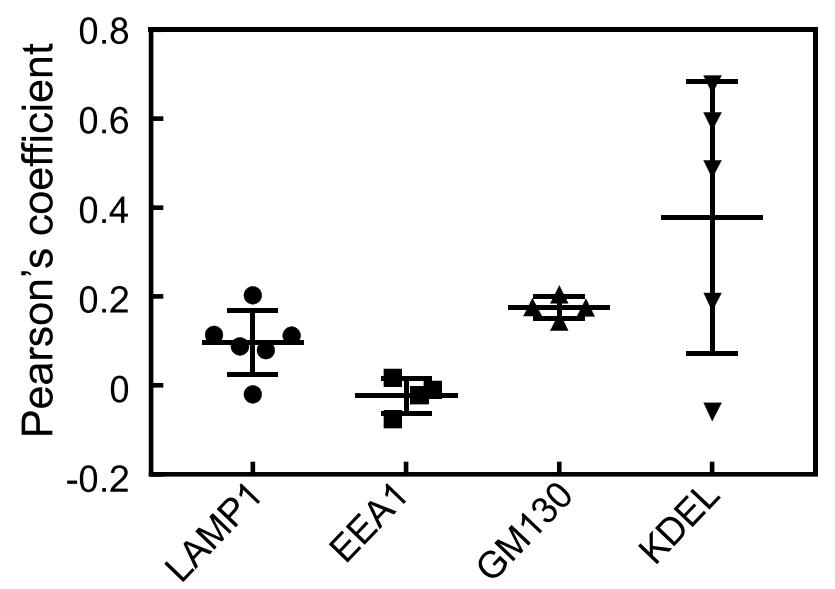

Figure 3. (continued) 


\section{$g \mathrm{hC} 1020 \mathrm{~A}$}
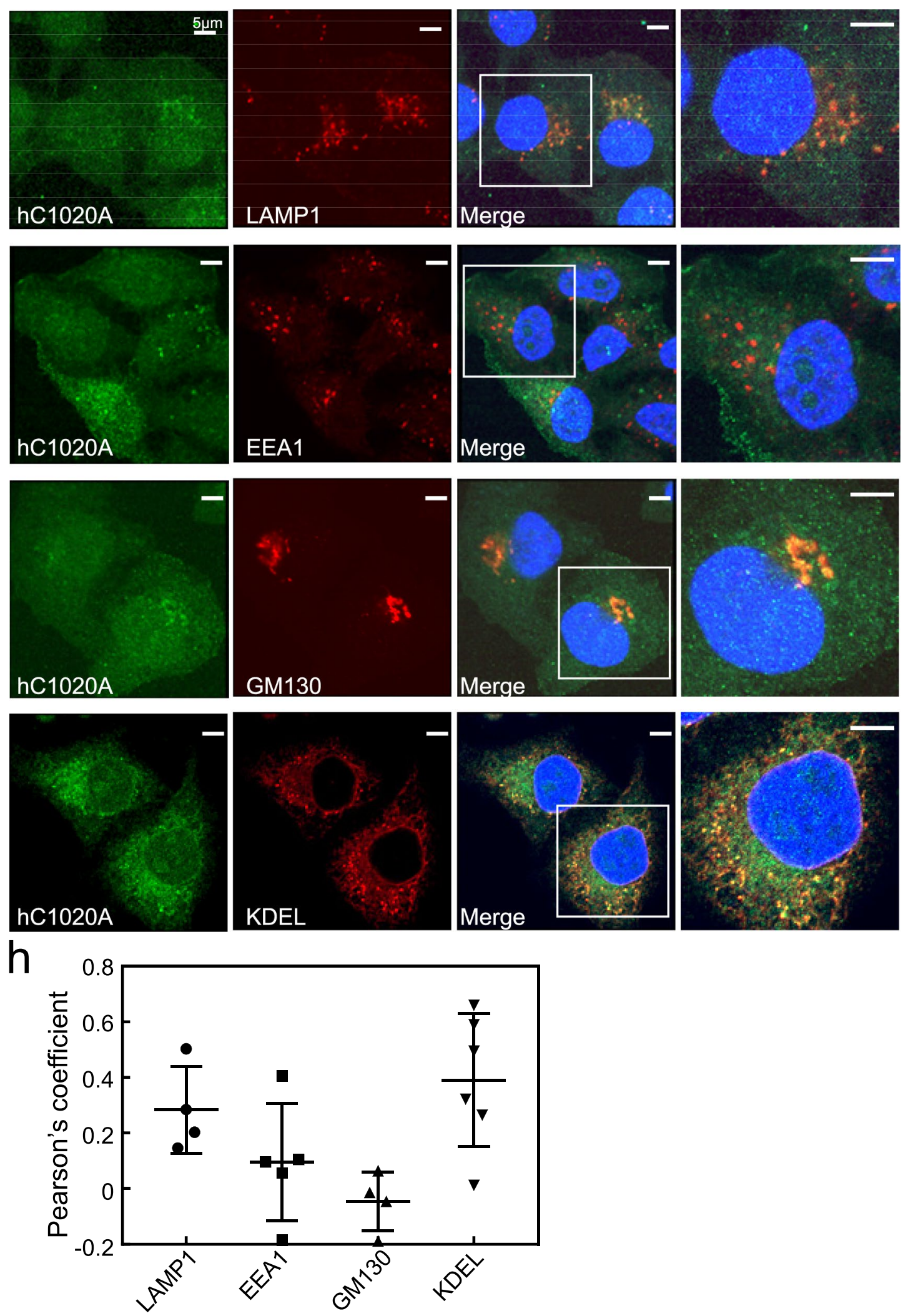

Figure 3. (continued) 


\section{i hC1019/1020A}
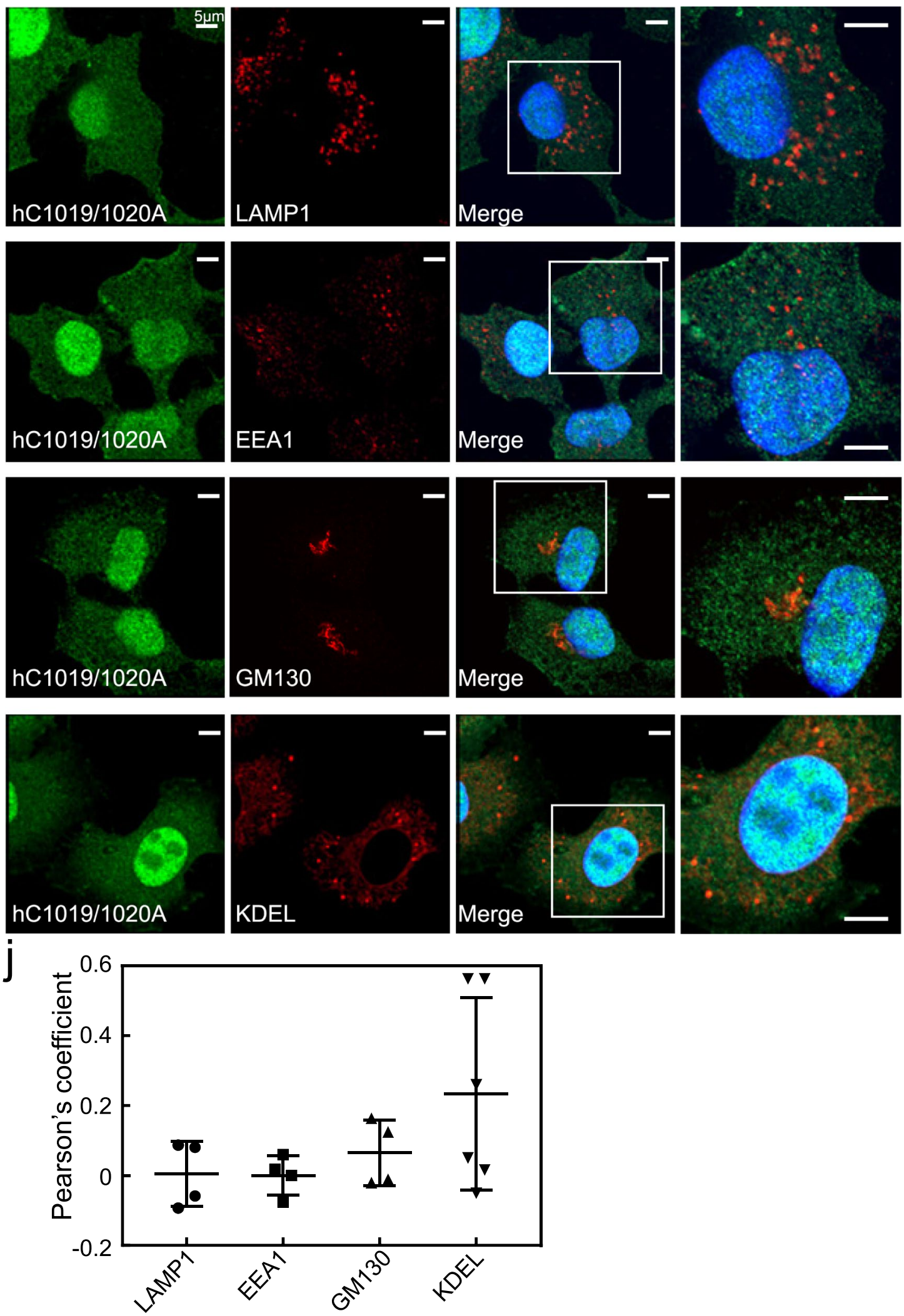

Figure 3. (continued) 


\section{a $\mathrm{h} \triangle \mathrm{EF}$}
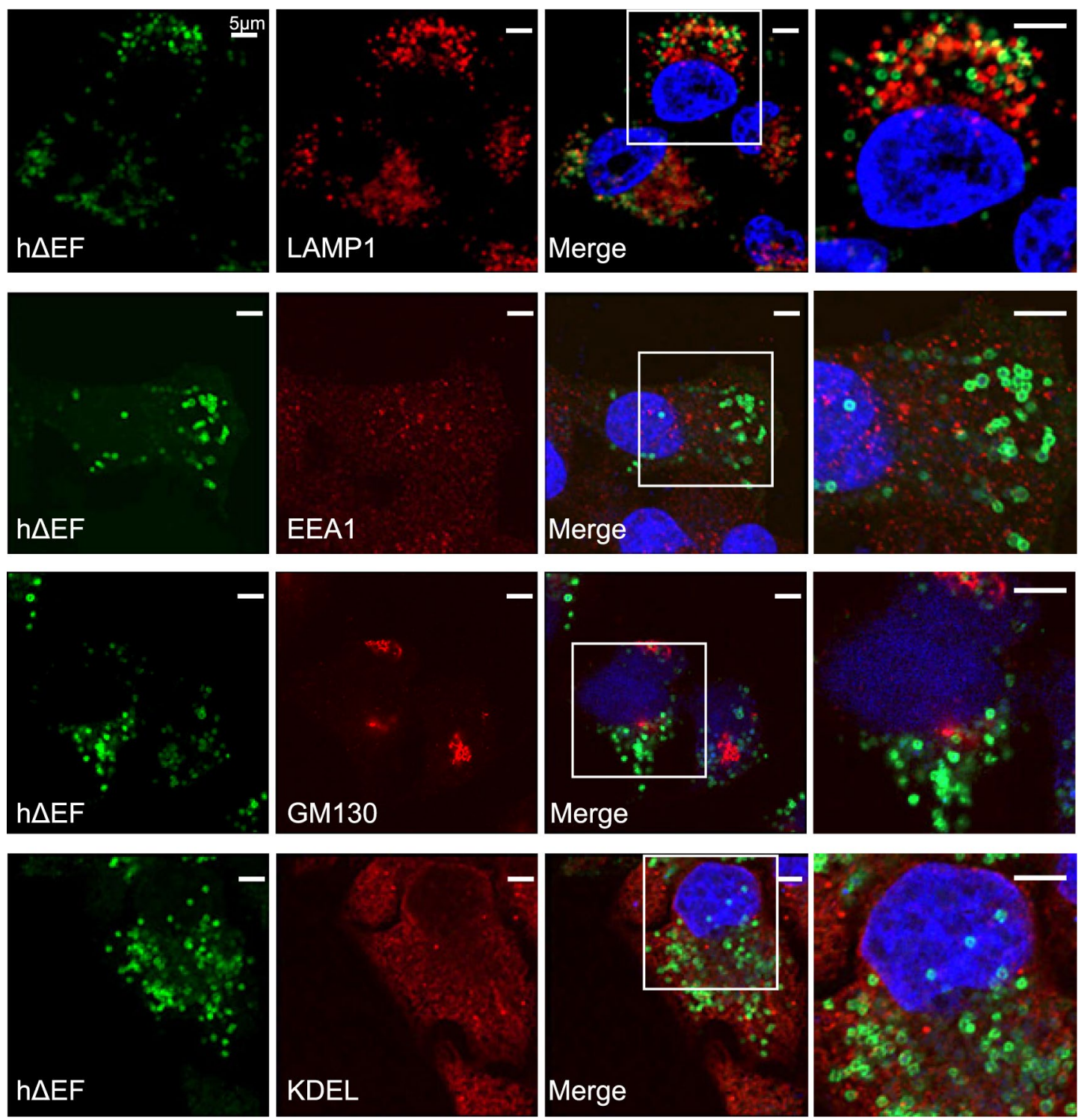

b

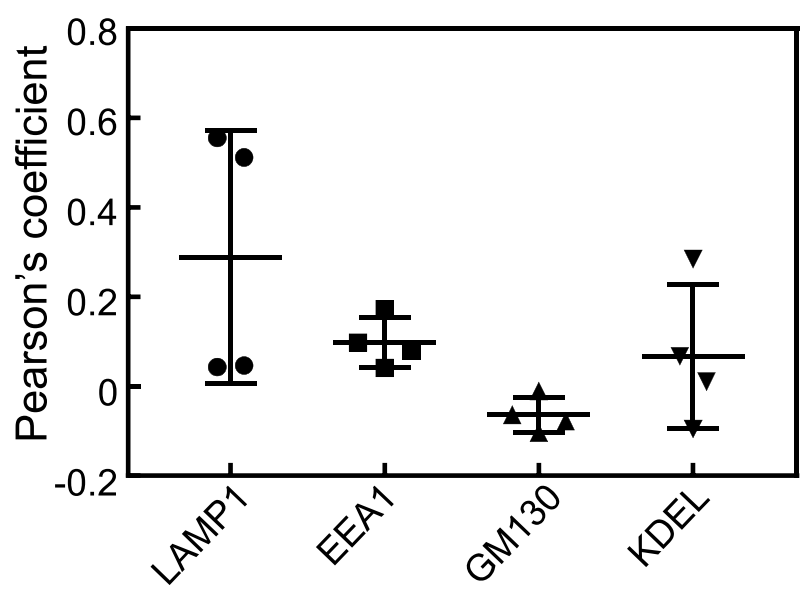

Figure 4. Subcellular localisation of the deletion mutants of hRab44 in HeLa cells. (a,b) eGFP-h $\Delta E F$, (c,d) eGFP-h $\Delta$ coil, and (e,f) eGFP-h826-1021. (a, c, e) Confocal laser microscopic analysis of HeLa cells immunofluorescently stained for LAMP1 (marker for late endosomes/lysosomes), EEA1 (marker for early endosomes), GM130 (marker for the Golgi), and KDEL (marker for ER). Bar: $5 \mu \mathrm{m}$. (b, d, f) Quantitative analysis of the ratio of colocalisation of these mutants with these organelle markers. Data are mean \pm SD $(n \geq 4)$. 


\section{C h $\Delta$ coil}
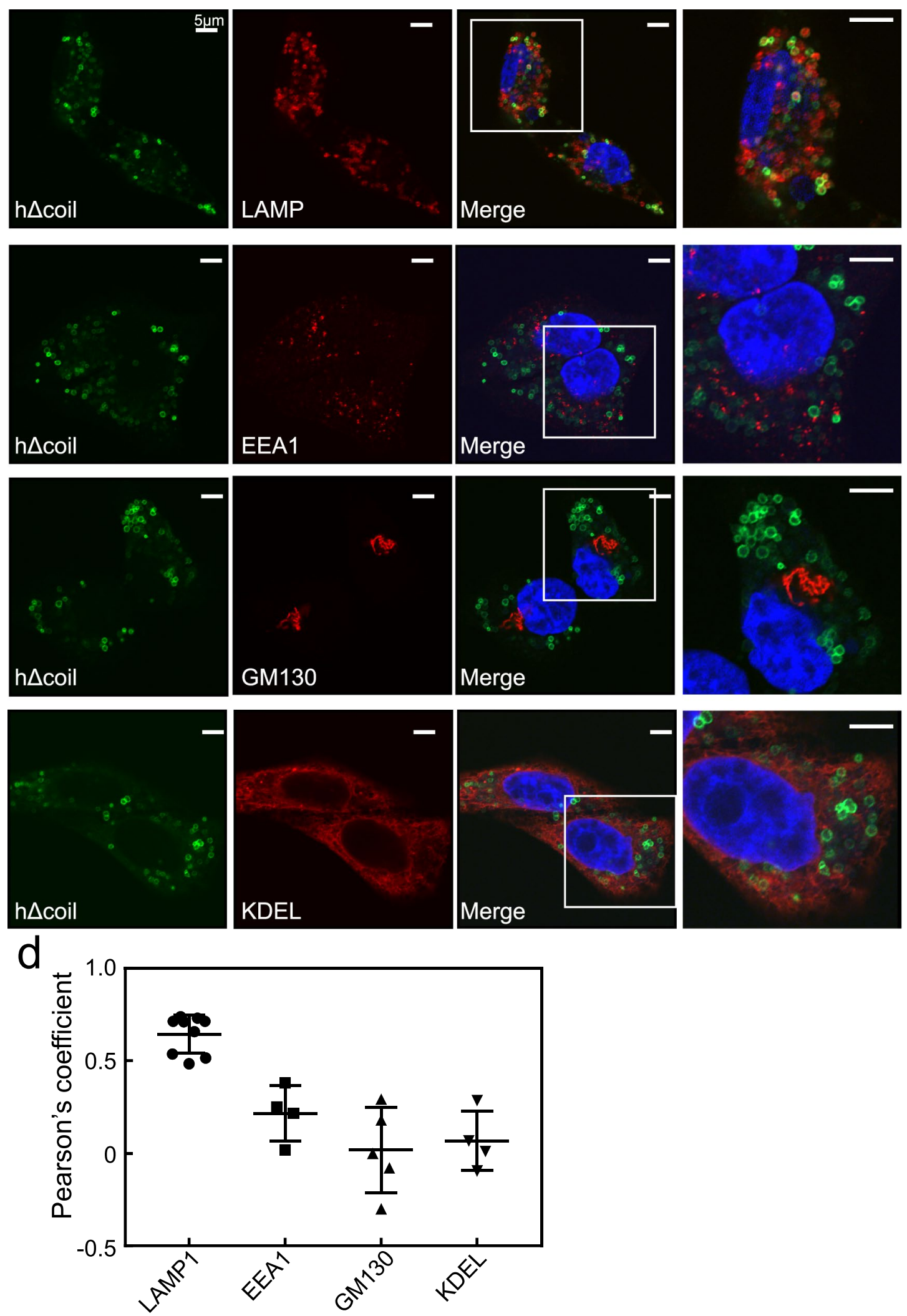

Figure 4. (continued) 
e h826-1021
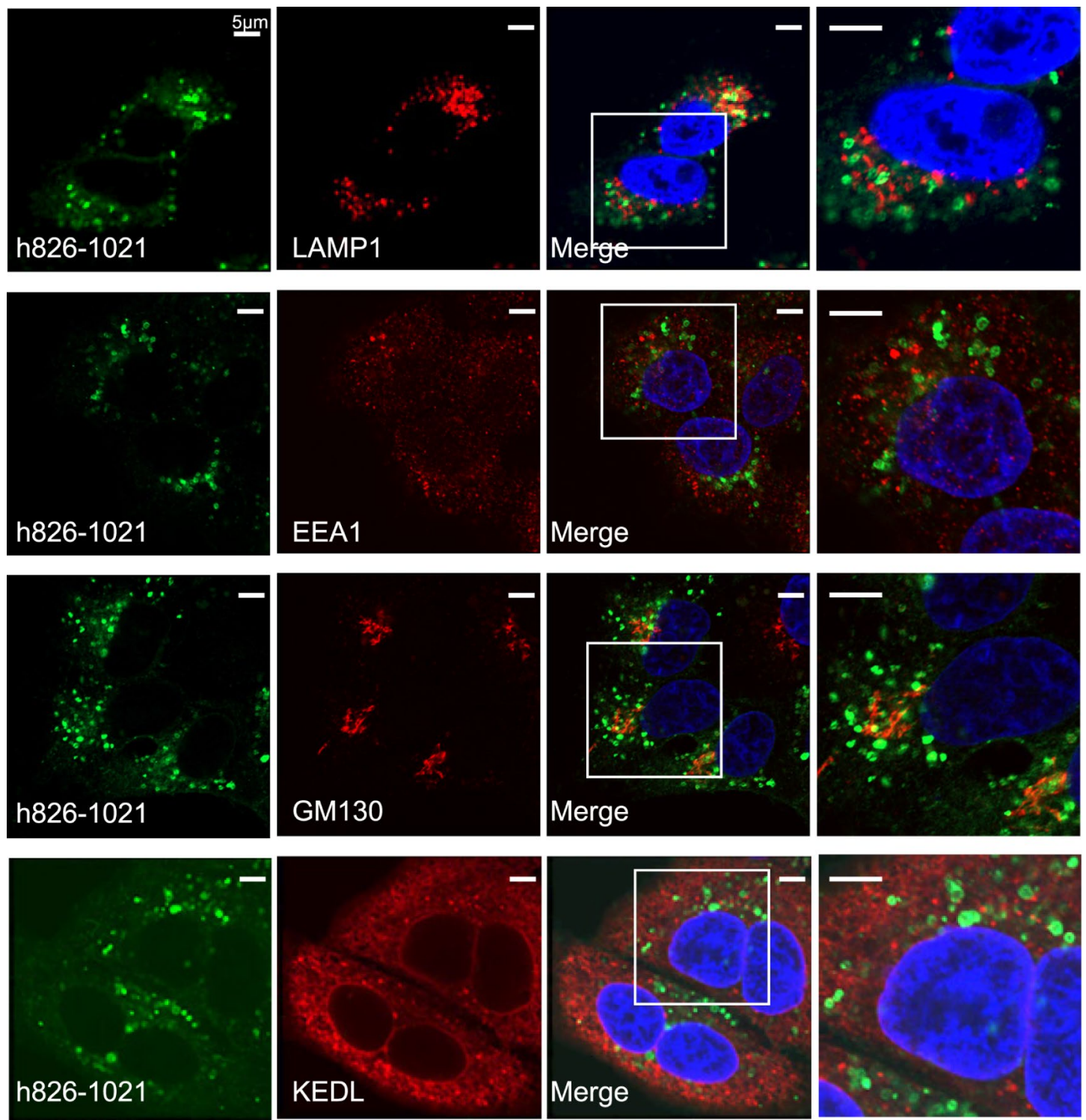

f

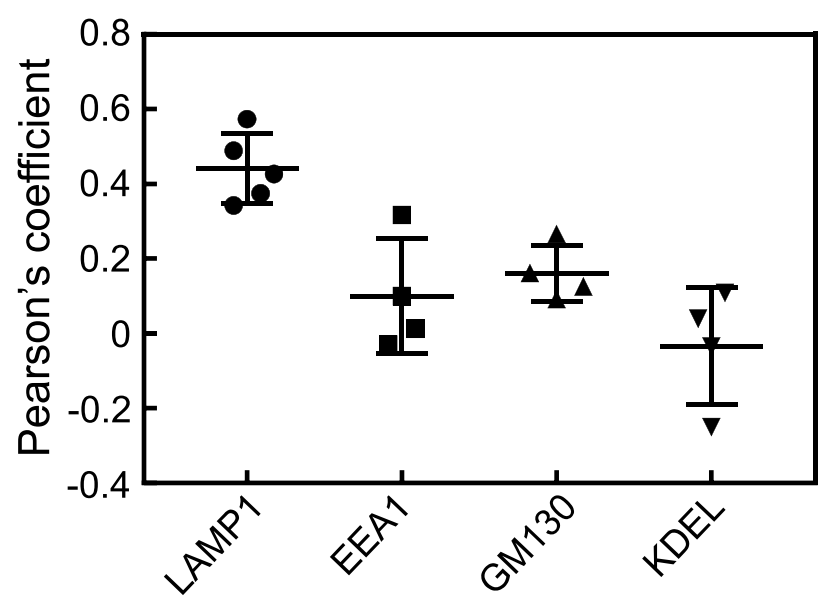

Figure 4. (continued) 

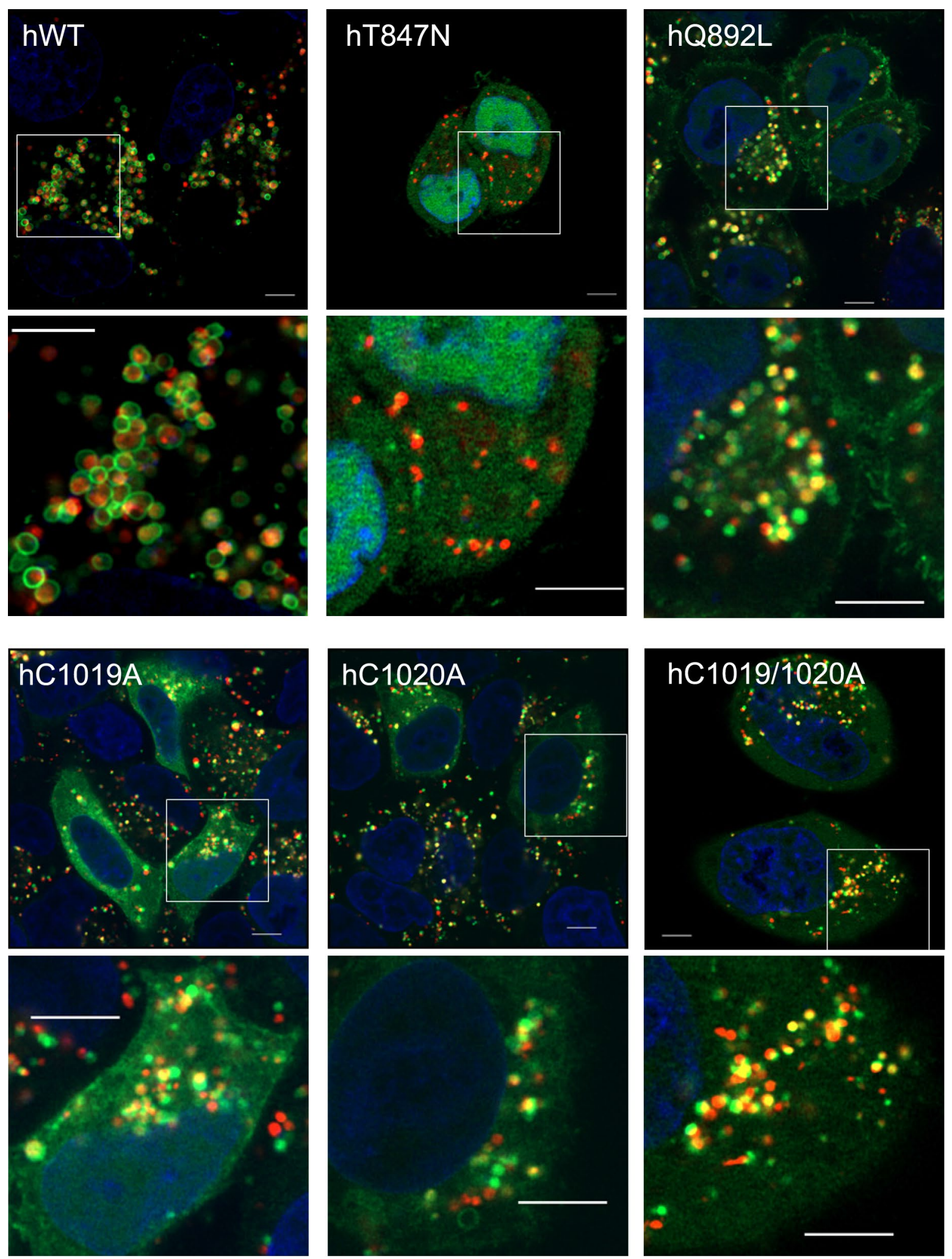

Figure 5. Subcellular localisation of LysoTracker in living HeLa cells expressing hWT and Rab44 mutants (eGFP-hT847N, eGFP-hQ82L, eGFP-hC1019A, eGFP-hC1020A, eGFP-hC1019/1020A, eGFP-h $\Delta$ EF, eGFP-h $\Delta$ coil, and eGFP-h826-1021). (a) Confocal laser microscopic analysis of live HeLa cells treated with LysoTracker-Red. Bar: $5 \mu \mathrm{m}$. (b) Size of LysoTrackerpositive vesicles in the live HeLa cells. The area of LysoTracker-positive vesicles was calculated using ImageJ, and the data were subjected to statistical analysis using 1-way ANOVA. Data are mean $\pm S D(n \geq 100)$. The asterisks indicate statistical significance compared to the hWT, ${ }^{\star} P<0.05,{ }^{* * *} P<0.001$, and ${ }^{* * *} P<0.0001$. 

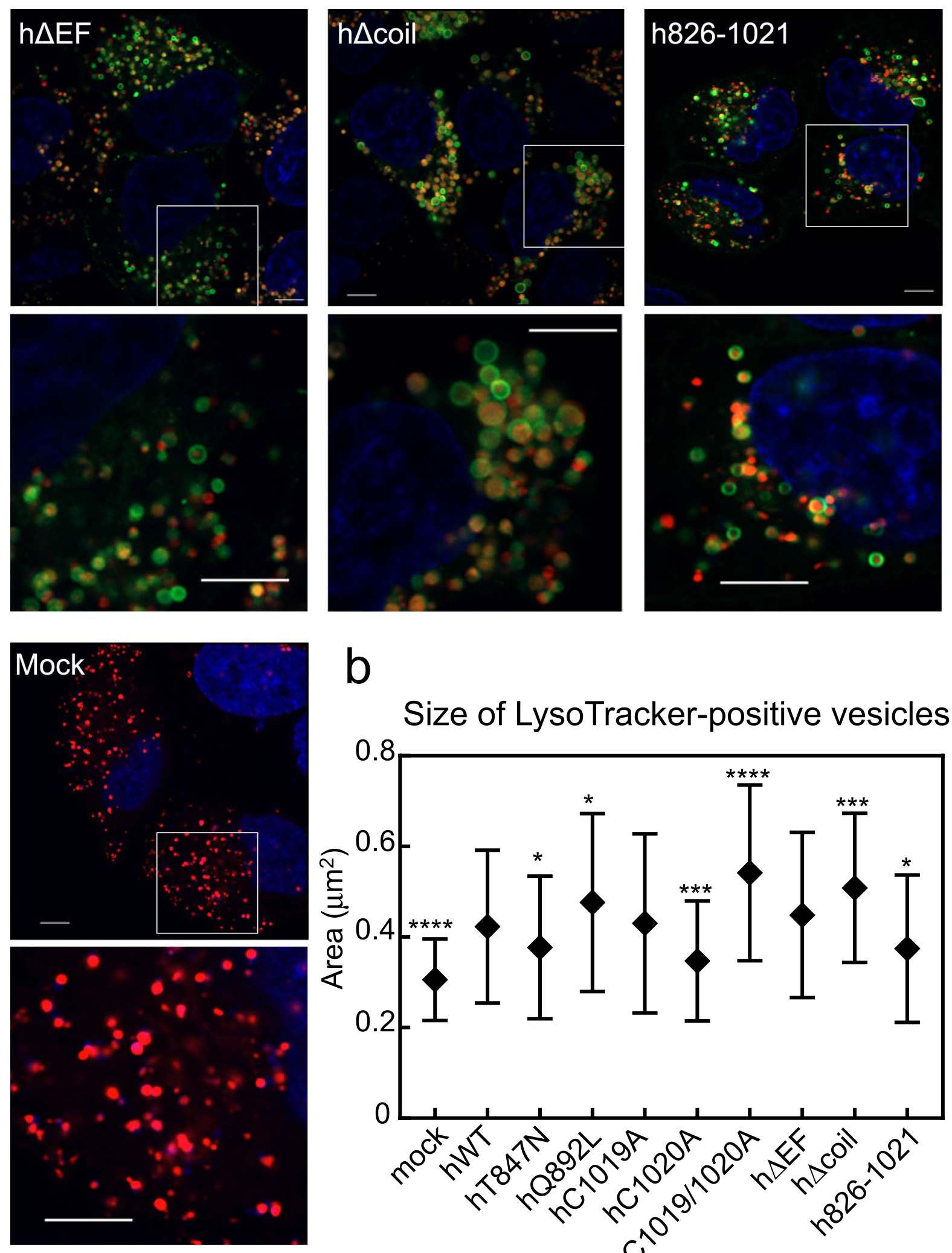

b

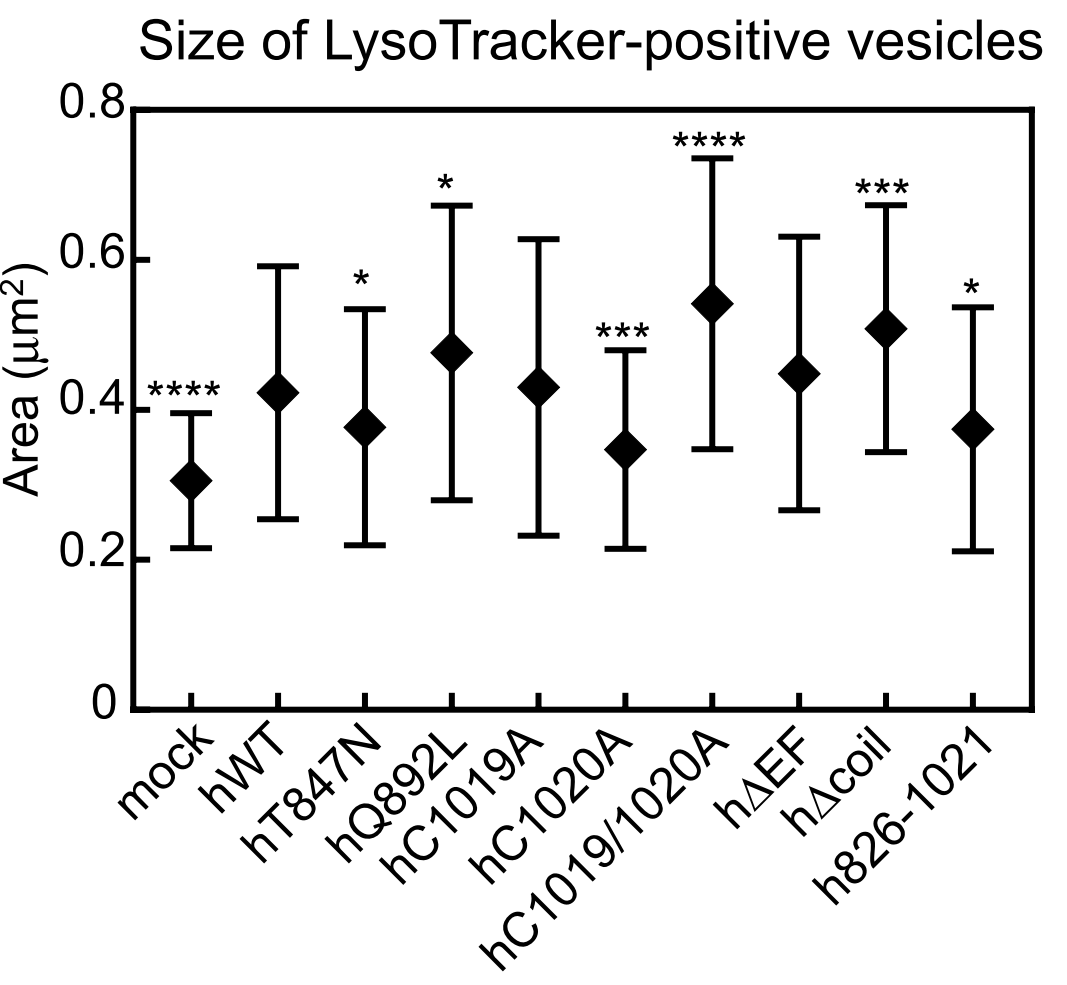

Figure 5. (continued) 

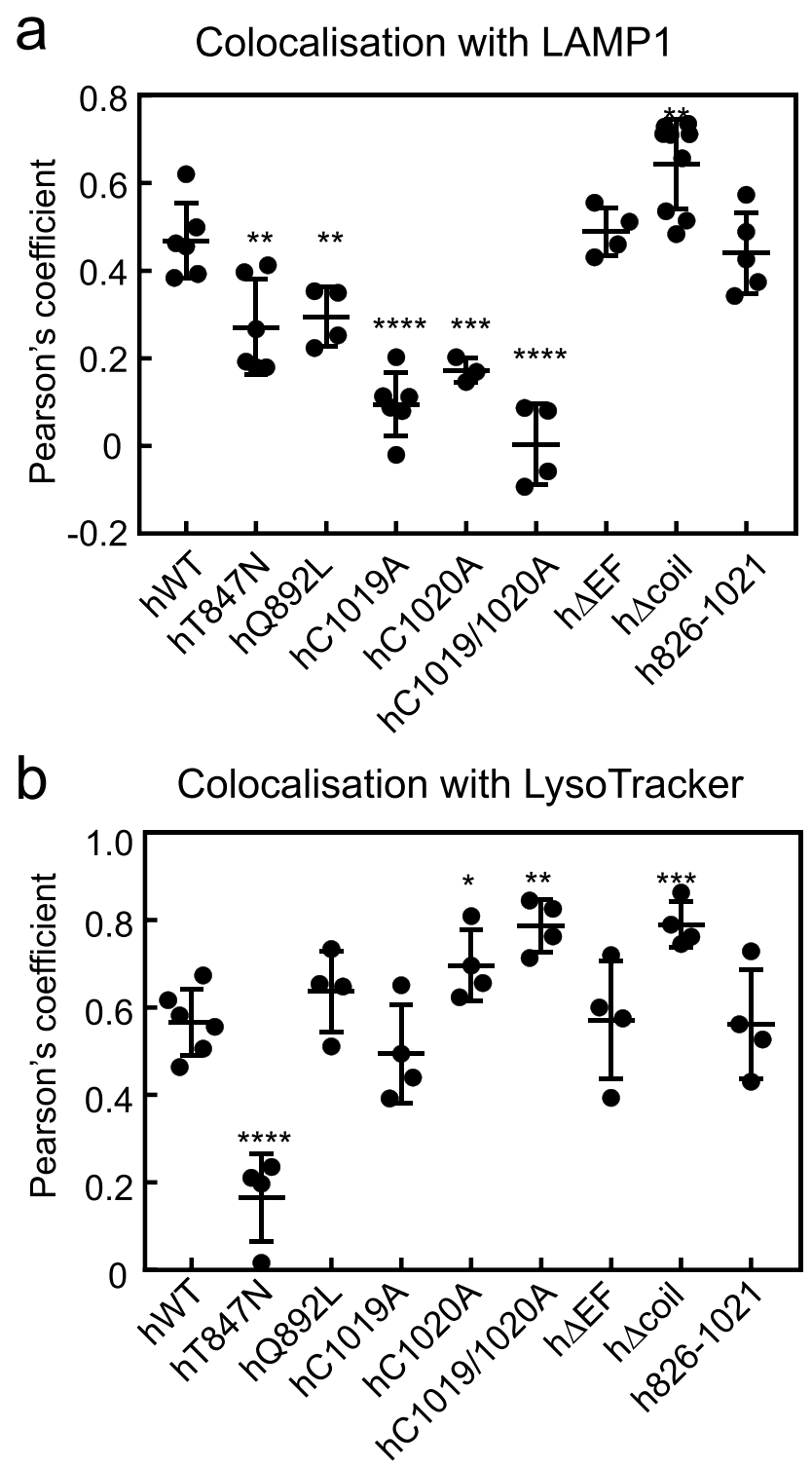

Figure 6. Comparing quantification of colocalisation of the hRab44 mutants with LAMP1 and LysoTracker. (a) Summary of quantitative analysis of colocalisation with LAMP1 (shown in Figs. 2, 3 and 4). (b) Quantitative analysis of colocalisation with LysoTracker. (shown in Fig. 5). The asterisks indicate statistical significance compared to the hWT, ${ }^{\star} P<0.05,{ }^{* *} P<0.01,{ }^{* *} P<0.001$, or ${ }^{* * * *} P<0.0001$.

and its mutants. Figure 5a shows the localisation pattern of hWT and its mutants in living cells stained with LysoTracker. Observations in live cells (Fig. 5a) were similar to those in fixed cells, as shown in Figs. 2, 3 and 4.

Quantitative analysis of the size of LysoTracker-positive compartments in live Hela cells are shown in Fig. $5 \mathrm{~b}$. hWT induced significantly larger LysoTracker-positive vesicles compared with control (Mock) (Fig. 5b). Interestingly, the DN mutant hT847N induced smaller LysoTracker-positive vesicles than hWT, while the CA mutant hQ892L induced larger LysoTracker-positive vesicles than hWT (Fig. 5b). The size of LysoTracker-positive vesicles was similar between hC1019A- and hWT-expressing cells (Fig. 5b). However, LysoTracker-positive vesicles were smaller in the C1020A-expressing cells and larger in C1019/1020A-expressing cells compared to those in hWT-expressing cells (Fig. 5b). Moreover, the h $\Delta \mathrm{EF}$ mutant yielded similar LysoTracker-positive vesicles to hWT. However, LysoTracker-positive vesicles were larger in $\mathrm{h} \Delta$ coil-expressing cells and smaller in h826-1021-expressing cells compared to those in hWT-expressing cells (Fig. 5b). These results indicate that the size of LysoTracker-positive vesicles is altered by Rab44 mutations (Fig. 5b).

Comparison of colocalisation of the human Rab44 mutants with LAMP1 and LysoTracker. Figure 6a shows a diagram summarizing quantitative analysis of colocalisation with LAMP1, supplementing the data shown in Figs. 2, 3 and 4. As compared with hWT, colocalisation with LAMP1 was significantly decreased for hT848N, hQ892L, hC1019A, hC1020A, and hC1019A/1020A mutants; significantly increased in the h $\Delta$ coil mutant; and unchanged in the $\mathrm{h} \triangle \mathrm{EF}$ and h826-1021 mutants (Fig. 6a). 
A quantitative analysis of colocalisation with LysoTracker is shown in Fig. 6b, supplementing the images in Fig. 5. When compared with the hWT, colocalisation with LysoTracker decreased only for the hT848N mutant, and increased for the hC1020A, hC1019/1020A, and h $\Delta$ coil mutants. The hQ892L, hC1019A, h $\Delta \mathrm{EF}$ and h8261021 mutants colocalised with LysoTracker at similar levels as hWT (Fig. 6b). Of particular note, differences between observations with LAMP1 and LysoTracker colocalisation analysis were found in mutants that are diffusely localised, such as hQ892L, hC1019A, hC1020A, and hC1019/1020A, suggesting that LAMP1 and LysoTracker detect different subcellular compartments.

Effects of $\mathrm{Ca}^{2+}$ modulators on localisation of human Rab44. Next, we assessed whether $\mathrm{Ca}^{2+}$ influx affects the subcellular localisation of human Rab44, since Rab44 encodes an EF-hand domain. As shown in Fig. 7, under basal conditions, hWT formed ring-like structures and partially surrounded the LAMP1-positive lysosomes in HeLa cells. However, when we treated hWT-expressing HeLa cells with ionomycin, a selective $\mathrm{Ca}^{2+}$ ionophore, the hWT partially translocated to small vesicles in the marginal region and to the plasma membrane and cytosol (Fig. 7a). We further examined the effects of other $\mathrm{Ca}^{2+}$-related reagents on the localisation of hWT. Upon treatment with thapsigargin, a $\mathrm{Ca}^{2+}$-ATPase inhibitor in the ER, hWT was detected in many vesicles distinct from LAMP1-positive lysosomes (Fig. 7a). ML-SA1 is a specific agonist for the lysosomal calcium channels transient receptor potential channel mucolipins (TRPML1-3). When hWT-expressing HeLa cells were treated with ML-SA1, the hWT localised to the plasma membrane and cytosol, and was also partially detectable in the lysosomes and non-lysosomal compartments (Fig. 7a). Following a quantitative analysis, we found that all the $\mathrm{Ca}^{2+}$ modulators significantly decreased colocalisation of hWT and LAMP1-positive lysosomes (Fig. 7b). These results indicate that transient $\mathrm{Ca}^{2+}$ influx induced by ionomycin or ML-SA1 causes partial translocation of hWT from the lysosomes to the plasma membrane and cytosol. In contrast, inhibition of intracellular $\mathrm{Ca}^{2+}$ with thapsigargin induces localisation of hWT to non-lysosomal vesicles.

Effects of ionomycin-mediated $\mathrm{Ca}^{2+}$ influx on localisation of various human Rab44 mutants. We further assessed whether ionomycin-mediated $\mathrm{Ca}^{2+}$ influx affects the subcellular localisation of human Rab44 and its mutants. Localisation of the DN mutant hT847N was unaffected by ionomycin treatment, and was partially detectable both in the cytosol and with LAMP1-positive lysosomes (Fig. 8a). Without stimulation, the CA mutant hQ892L was detected in LAMP1-positive lysosomes surrounded by ring-like structures and partially in the plasma membrane (Fig. 8b). However, following ionomycin treatment, the formation of ring-like structures surrounding the LAMP1-positive lysosomes was slightly enhanced - though the difference was not statistically significant - and its localisation to the plasma membrane decreased (Fig. 8b). Importantly, the h $\Delta \mathrm{EF}$ mutant formed ring-like structures even after ionomycin treatment, and its localisation was nearly unchanged by stimulation (Fig. 8c). Therefore, the EF-hand domain is likely to be important for $\mathrm{Ca}^{2+}$-mediated localisation of human Rab44. The $\mathrm{h} \Delta$ coil mutant localised partially to lysosomal and non-lysosomal vesicles without ionomycin, and was partially localised to the cytosol with ionomycin treatment (Fig. 8d). Ionomycin treatment significantly reduced the colocalisation ratio of the $\mathrm{h} \Delta$ coil mutant with LAMP1-positive lysosomes (Fig. 8f). Ionomycin had no effect on the localisation of the h826-1021 mutant to non-lysosomal vesicles or its partial colocalisation with LAMP1-positive lysosomes (Fig. 8e). Quantification of the effects of ionomycin on colocalisation of Rab44 mutants with LAMP1-positive lysosomes is shown in Fig. 8f. Ionomycin treatment significantly reduced the colocalisation of hWT or h $\triangle$ coil mutant with LAMP1-positive lysosomes, but had little effect on the mutants lacking the EF-hand domain, such as the $\mathrm{h} \Delta \mathrm{EF}$ and h826-1021 (Fig. 8f). Thus, the localisation of Rab44 is altered by ionomycin, and the EF-hand domain of human Rab44 is important for $\mathrm{Ca}^{2+}$-mediated regulation of Rab44 localisation.

\section{Discussion}

In this study, we constructed various mutants of human Rab44 using site-directed mutagenesis. Wild-type human Rab44 (hWT) and the Rab44 mutant constructs were expressed exogenously in HeLa cells.

The expression level of the h826-1021 mutant was extremely low, for which there may be a few possible causes. First, whereas the wild-type protein contains 1021 amino acid residues, the h826-1021 is reduced to $19 \%$ of its original size with only 196 amino acid residues. Second, this fragment alone may be unstable as a protein. In other words, the N-terminal domains may be important for stable formation of the protein, even though the structure of this mutant is similar to that of other small Rab proteins.

Upon analysing the DN and CA mutants, Rab44 was found to share some common features with other small Rab GTPases. In many small Rab GTPases, differences in localisation have been observed between the DN and CA mutants. For example, when human Rab21 was overexpressed in HeLa cells, it predominantly localised to EEA1-containing early endosomes. The DN mutant Rab21(T33N) was concentrated in the perinuclear region, whereas the CA mutant Rab21(Q78L) localised to the reticular tubular network ${ }^{20}$. Therefore, it is possible that differences in localisation between the DN mutant hT847N and CA mutant hQ892L affect cell shape: hT847N caused a round cellular shape with clear contacts between cells, whereas hQ892L resulted in unclear cell-cell contact sites.

The lipidation-site mutants of Rab44 also shared some common features with those of the small Rab GTPases. The prenylatable cysteines are key factors for membrane targeting of Rab GTPases. For example, lipidation-site mutations in both Rab4 and Rab5 cause complete cytosolic localisation ${ }^{21}$. Similarly, the lipidation-site mutant, hC1019A, was mostly localised to the cytosol and partially to lysosomes (Fig. 3c), whereas the lipidation-site mutants hC1020A and hC1019/1021A completely localised to the cytosol (Fig. 3d,e). Interestingly, hC1019A partially localised to LAMP1-positive lysosomes, whereas hC1020A was mostly diffusely distributed throughout the cytoplasm. Therefore, hC1020A appears to have a greater effect on subcellular localisation than hC1019A. 

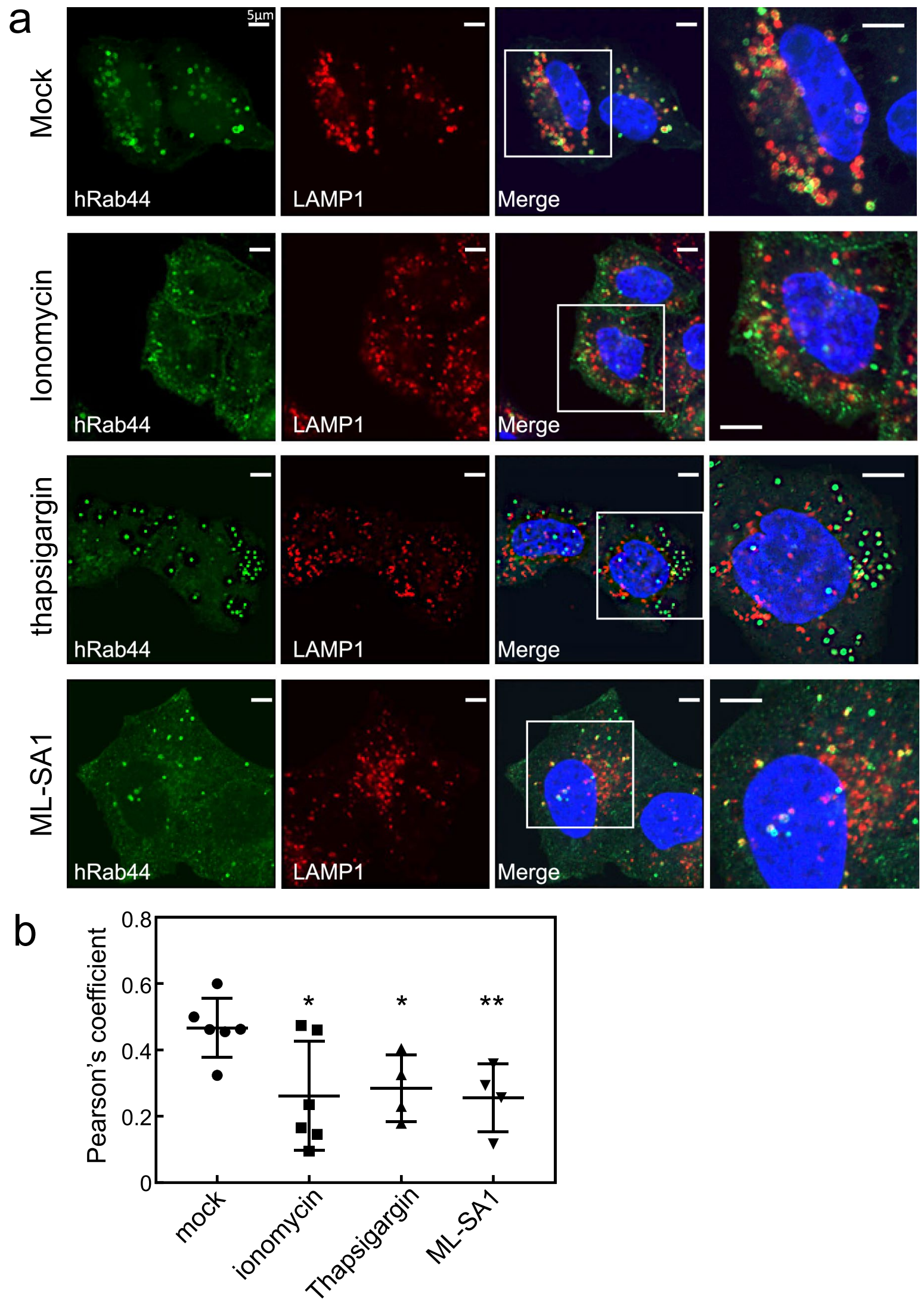

Figure 7. Effect of $\mathrm{Ca}^{2+}$ modulators on localisation of human Rab44 (hWT) expressed in HeLa cells. Human Rab44 (hWT)-expressing HeLa cells were treated with mock, ionomycin, thapsigargin, or ML-SA1. (a) Confocal laser microscopic analysis of HeLa cells immunofluorescently stained for LAMP1 (marker for late endosomes/ lysosomes). Bar: $5 \mu \mathrm{m}$. (b) Quantitative analysis of the ratio of colocalisation of hWT with LAMP1. 


\section{a $\mathrm{hT} 847 \mathrm{~N}(\mathrm{DN})$}
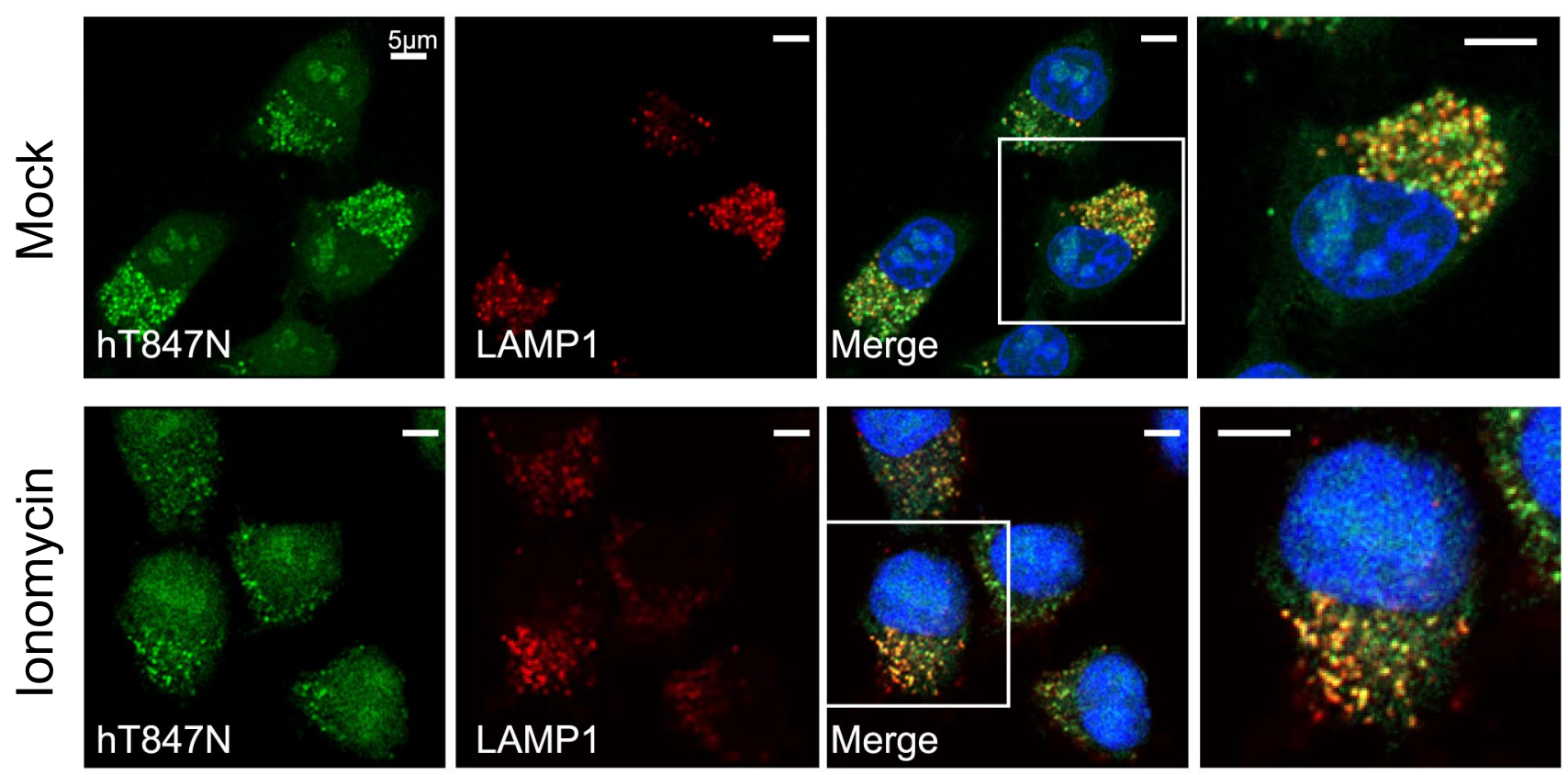

b
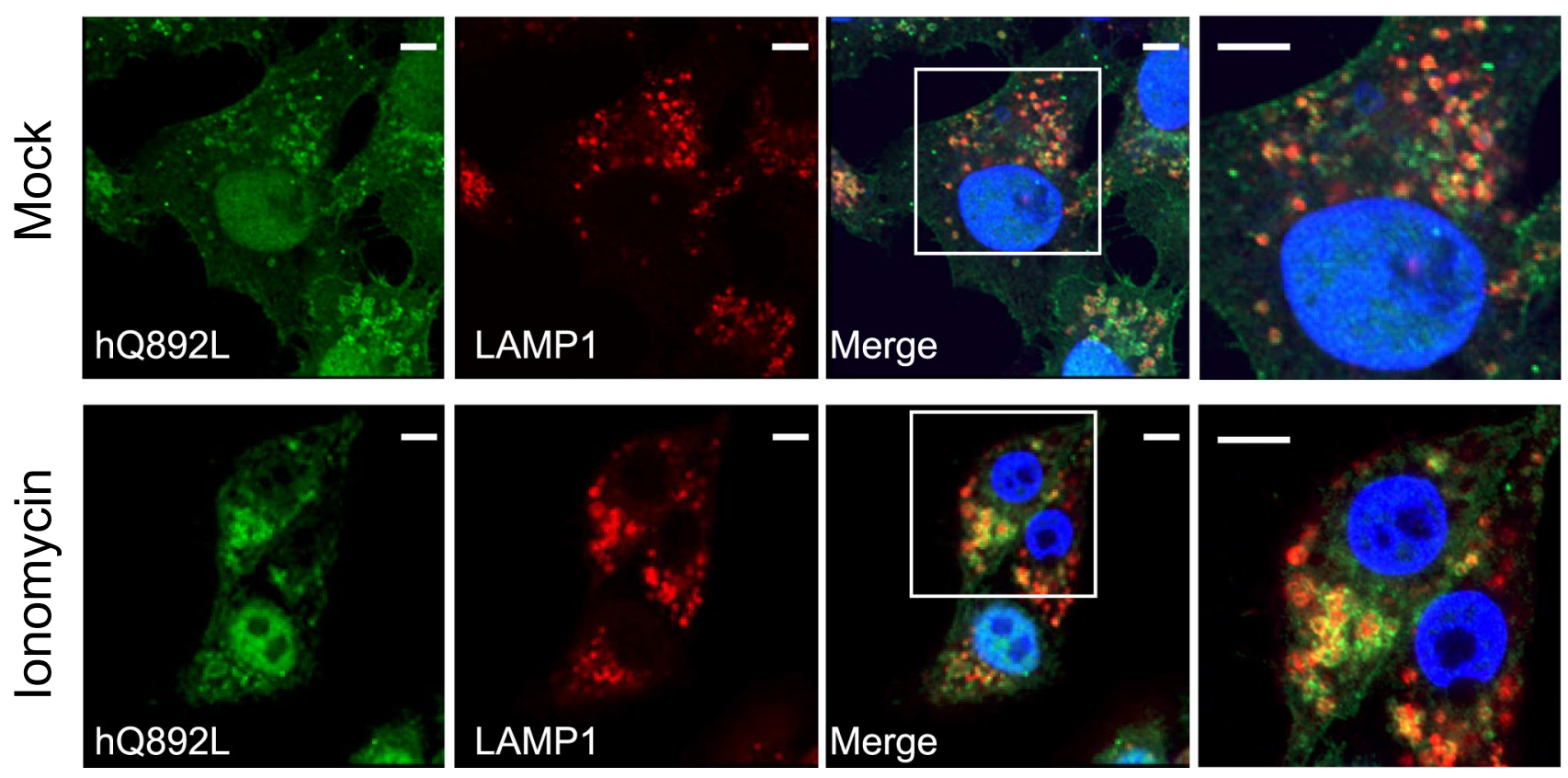

Figure 8. Effect of ionomycin on localisation of mutants of human Rab44 expressed in HeLa cells. (a) eGFPhT847N (DN), (b) eGFP-hQ892L (CA), (c) eGFP-h $\Delta$ EF, (d) eGFP-h $\Delta$ coil, and (e) eGFP-h826-1021 in HeLa cells. Human Rab44 (hWT)-expressing HeLa cells were treated with mock, or ionomycin. Confocal laser microscopic analysis of HeLa cells immunofluorescently stained for LAMP1 (marker for late endosomes/ lysosomes). Bar: $5 \mu \mathrm{m}$. (f) Quantitative analysis of the colocalisation of the indicated Rab44 mutants with LAMP1 with or without ionomycin treatment. 


\section{C $\mathrm{h} \triangle \mathrm{EF}$}
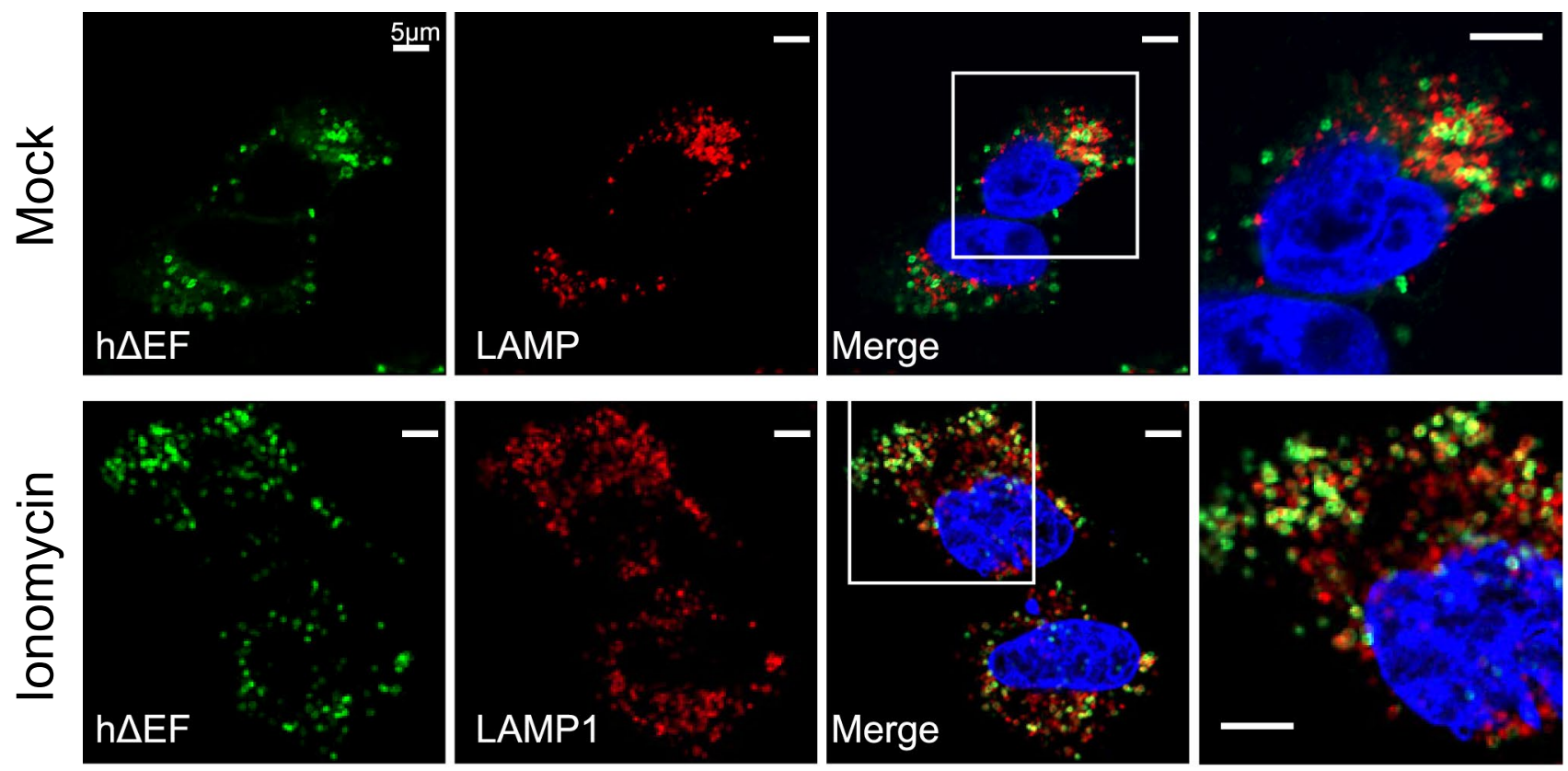

d $\mathrm{h} \Delta$ coil
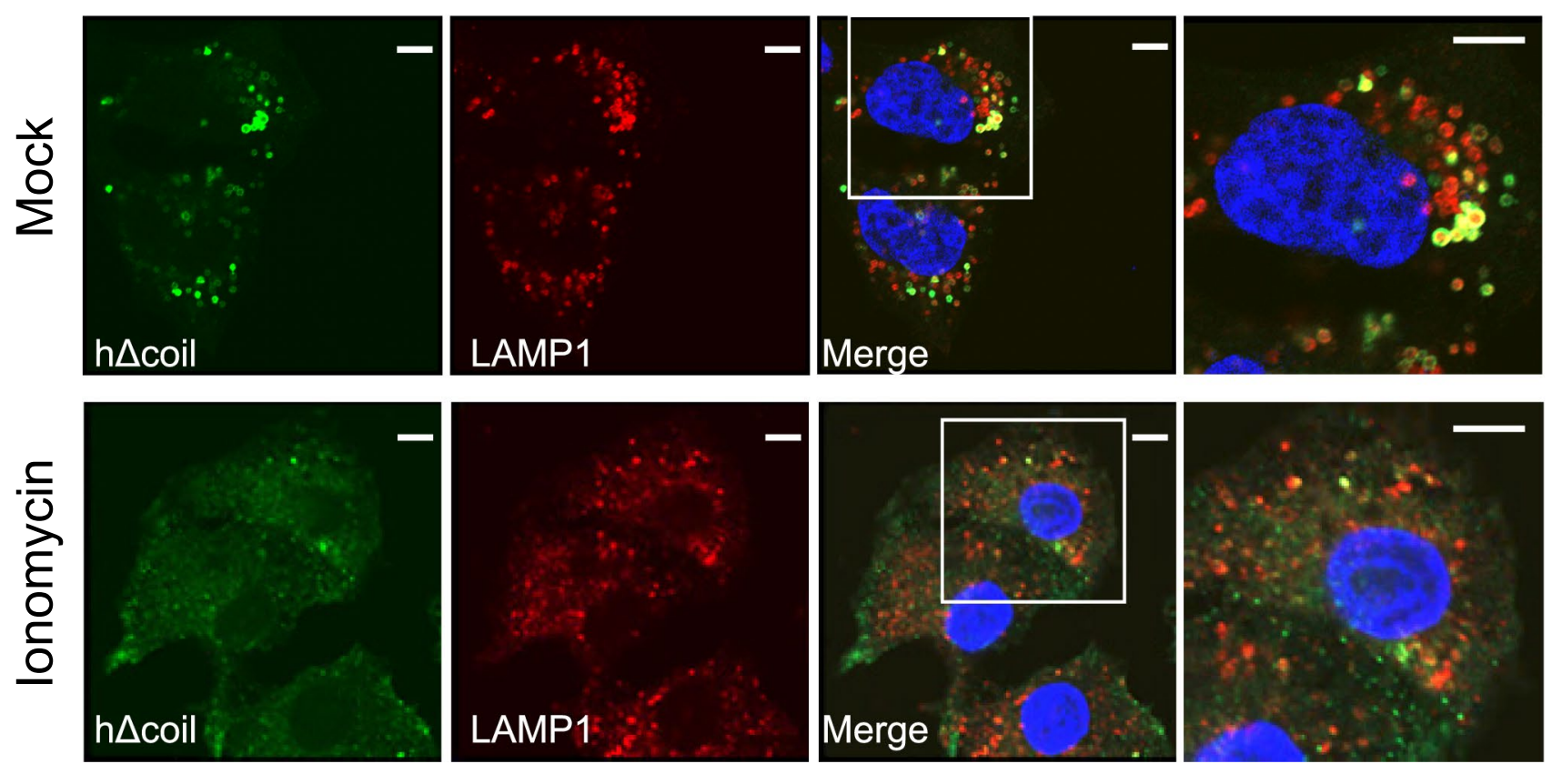

Figure 8. (continued) 


\section{e h826-1021}
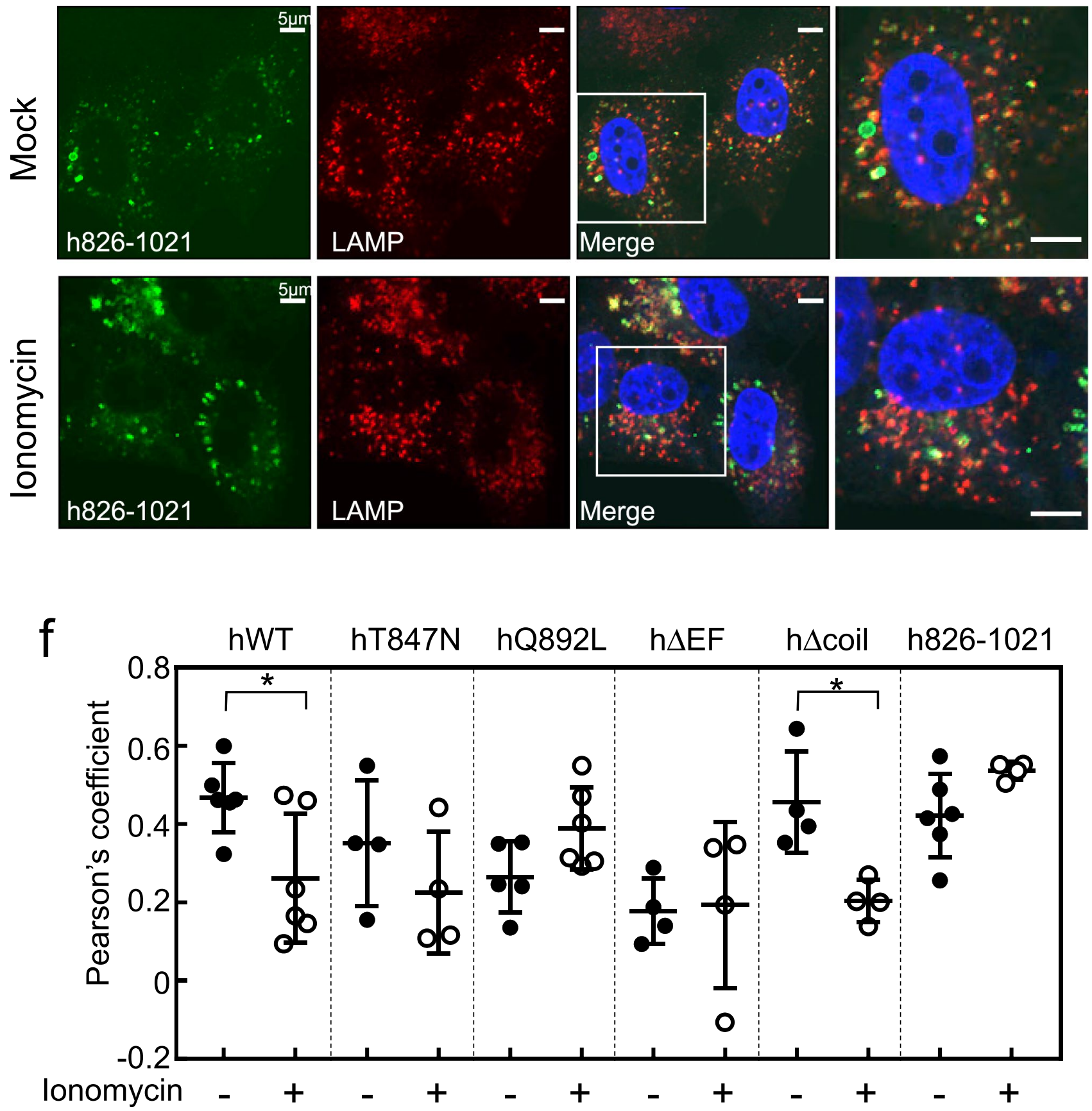

Figure 8. (continued)

The EF-hand domain, which is known to be the $\mathrm{Ca}^{2+}$ binding site ${ }^{22}$, is likely to be required for the partial translocation of Rab44 into the plasma membrane and cytosol under conditions of transient $\mathrm{Ca}^{2+}$ influx. Consistent with this notion, transient $\mathrm{Ca}^{2+}$ influx caused partial translocation of the constructs containing the EF-hand domain, including $\mathrm{hWT}$ and $\mathrm{h} \Delta$ coil, into the plasma membrane and cytosol. Considering that the CA mutant, hQ892L, partially localised to the plasma membrane and cytosol without stimulation, the EF-hand domain of Rab44 may be an important activation factor for translocation induced by $\mathrm{Ca}^{2+}$ mobilisation. In contrast, translocation of constructs lacking the EF-hand domain, including $\mathrm{h} \Delta \mathrm{EF}$ and h826-1021 mutants, were virtually unaffected by ionomycin-mediated transient $\mathrm{Ca}^{2+}$ influx conditions. Taken together, the EF-hand domain of Rab44 is probably important for $\mathrm{Ca}^{2+}$-mediated translocation.

The coiled-coil domain appears to be important for localisation and formation of LysoTracker-positive vesicles as described below. Given that mutants lacking the coiled-coil domain, such as h $\Delta$ coil and h826-1021 mutants, had a higher colocalisation coefficient with LAMP1-positive lysosomes and a lower one with KDEL-positive 
compartment compared with hWT, it is likely that fusion of the mutants lacking the coiled-coil domain with lysosomes is increased but fusion with the ER is decreased. Generally, the coiled-coil domain-containing proteins have been proposed to function as tethering factors to target organelles prior to fusion or as scaffolds for the assembly of other factors important for fusion ${ }^{23,24}$. Therefore, it would be interesting to investigate the molecular mechanisms through which the Rab44 coiled-coil domain assists in localisation and organelle formation.

The size of LysoTracker-positive vesicles was affected by Rab44 expression and mutation. hWT induced larger vesicles compared to the control, the DN mutant hT847N induced smaller vesicles than the WT, and the CA mutant hQ892L induced larger vesicles than hWT; therefore, it is likely that Rab44 has effects on a large formation of LysoTracker-positive vesicles. Moreover, it is interesting that the combination of hC1020A and hC1019/1020A mutants and the combination of $\mathrm{h} \Delta$ coil and h826-1021 mutants had the exact opposite effects on formation of LysoTracker-positive vesicles, although the mechanisms behind these effects remain unknown.

Interestingly, differences in colocalisation between LAMP1 and LysoTracker were observed in the mutants that are diffusely localised, such as hQ892L, hC1019A, hC1020A, and hC1019/1020A (Fig. 6). Specifically, these mutants had decreased colocalisation with LAMP1, and increased colocalisation with LysoTracker compared to hWT. There are known to be some differences between LAMP1-positive compartments and LysoTrackerstained organelles ${ }^{25}$. Therefore, we speculate that LysoTracker may detect LAMP1-negative acidic vesicles. Indeed, LysoTracker has reported to be an acidotropic dye that stains intermediate compartments, including autolysosomes, and phagolysosomes as well as late endosomes/lysosomes ${ }^{26,27}$.

Ionomycin- and ML-SA1-induced transient $\mathrm{Ca}^{2+}$ influx caused partial translocation of hWT from the lysosomes into the plasma membrane and cytosol. In contrast, inhibition of intracellular $\mathrm{Ca}^{2+}$ using thapsigargin induced translocation of hWT to non-lysosomal vesicles. Thus, the translocation of Rab44 is regulated by the intracellular $\mathrm{Ca}^{2+}$ level, and the EF-hand domain of $\mathrm{Rab} 44$ is important for the $\mathrm{Ca}^{2+}$-mediated translocation.

In conclusion, the roles of the EF-hand, coiled-coil domains, and lipidation sites in human Rab44 were analysed using deletion and point mutants. The EF-hand domain is required for partial translocation of Rab44 into the plasma membrane and cytosol under conditions of transient $\mathrm{Ca}^{2+}$ influx, and the coiled-coil domain is important for localisation, formation of LysoTracker-positive vesicles. The lipidation-site is essential for localisation to the membrane.

\section{Materials and methods}

Antibodies and reagents. Mouse monoclonal anti-GAPDH (Cat. No. M171-3), anti-GFP (Cat. No. 598), anti-EEA1 (Cat. No. M176-3), and anti-KDEL (Cat. No. M181-3) antibodies were purchased from Medical \& Biological Laboratories (Nagoya, Japan). Mouse monoclonal anti-mouse LAMP1 (Cat. No. 555798) and anti-GM130 (Cat. No. 610823) antibodies were from BD Biosciences (Franklin Lakes, NJ, USA). Alexa Fluor 488-conjugated goat anti-rabbit IgG and Alexa Fluor 555-conjugated goat anti-mouse, anti-rat IgG, and antirabbit IgG were from ThermoFisher Scientific (Rockford, IL, USA). Ionomycin, thapsigargin, and ML-SA1 were purchased from FUJIFILM-WAKO (Osaka, Japan).

Cell culture. HeLa cells were grown in Dulbecco's modified Eagle's medium (DMEM) containing $10 \%$ fetal bovine serum, $50 \mathrm{U} / \mathrm{mL}$ penicillin, and $50 \mu \mathrm{g} / \mathrm{mL}$ streptomycin at $37^{\circ} \mathrm{C}$ in a $5 \% \mathrm{CO}_{2}$ atmosphere.

Retrovirus construction and expression of Rab44 and mutants. The full-length human Rab44 gene was synthesised and cloned into pcDNA3.1+N-DYK using GenScript (Piscataway, NJ, USA). The Rab44 gene mutants were generated using polymerase chain reaction (PCR) using a PrimeSTAR mutagenesis kit (Takara, Shiga, Japan). The primers used for PCR were; for eGFP (for In-Fusion) Forward: GAATTAGATCTCTCGAGA TGGTGAGCAAGGGCGAGGA and Reverse: TCTCTGTCCAGTCTCCTTGTACAGCTCGTC; Rab44 WT Forward: GGACGAGCTGTACAAGGAGACTGGACAGAGA, and Reverse: AATTCGTTAACCTCGAGT CAGGAGCAACAGCCG; hQ892L Forward: CAGCTGGCCTTGAGAGGTACCACAGTATG, and Reverse: TACCTCTCAAGGCCAGCTGTGTCCCAGAG; hT847N Forward: GGCAAAAACTCCTTCCTGCACCTG CTG, and Reverse: GAAGGAGTTTTTGCCCACGTTGGAGTC; hC1019A Forward: TTCGGCGCTTGCTCC TGACTCGAGGTTA, and Reverse: AGGAGCAAGCGCCGAATCTCTTGGGCG G; hC1020A Forward: GGC TGTGCTTCCTGACTCGAGGTTAAC, and Reverse: TCAGGAAGCACAGCCGAATCTCTTGGG; hC1019A/ C1020A Forward: GGCGCTGCTTCC TGACTCGAGGTTAAC, and Reverse: TCAGGAAGCAGCGCCGAA TCTCTTGGG; $\triangle$ EF Forward: CTCTCAGAGGAAGCCACTGCCCTCT, and Reverse: GGCTTCCTCTGA GAGGACCAAGACTC; $\triangle$ coil Forward: CTTCATGCAGCTACTGAGCAACTTT, and Reverse: GTAGCTGCA TGAAGGCAAGGAACGC; and h826-1021 Forward: TGTACAAGCCCCAGGCCAACCCTGAT, and Reverse: GCCTGGGGCTTGTACAGCTCGTCCAT.

The PCR products were cloned into the retroviral vector, pMSCVpuro (Clontech, Mountain View, CA, USA), using an In-Fusion cloning kit (Clontech). The pMSCVpuro vector with an eGFP fragment insert yielding N-terminal eGFP-fusion proteins was kindly provided by Prof. Kosei Ito (Nagasaki University, Japan). The vectors were transfected into HEK293T cells using Lipofectamine 3000 (Life Technologies, Gaitherburg, MD, USA), according to the manufacturer's instructions. After incubation at $37^{\circ} \mathrm{C}$ in a $5 \% \mathrm{CO}_{2}$ atmosphere for $48 \mathrm{~h}$, the supernatants containing the viral particles were collected and used to infect HeLa cells. Cells stably expressing Rab44 were selected using puromycin $(5 \mu \mathrm{g} / \mathrm{mL})$ in the culture medium, and media was changed every third day after 3 weeks.

Western blot analysis. Western blotting analysis was performed according to the protocol described previously ${ }^{28-30}$. Briefly, the cells were lysed in cell lysis buffer supplemented with protease inhibitors. Equal amounts of protein were subjected to sodium dodecyl sulphate polyacrylamide gel electrophoresis (SDS-PAGE) 
followed by transfer onto a polyvinylidene difluoride (PVDF) membrane. The blots were blocked with $5 \%$ milk in Tris-buffered saline (TBS) for $1 \mathrm{~h}$ at $25^{\circ} \mathrm{C}$, incubated with anti-GFP antibody (1:3000) for $2 \mathrm{~h}$ at $4{ }^{\circ} \mathrm{C}$ and washed four times with TBS containing $0.1 \%$ Tween 20 . The blots were then incubated with horseradish peroxidase-conjugated secondary antibodies for $1 \mathrm{~h}$ at $25^{\circ} \mathrm{C}$, washed, and detected with Immobilon Forte ECL HRP substrate (Merck-Millipore, Burlington, MA, USA). Immunoreactive bands were analysed using a LAS-4000 Mini imaging system (Fujifilm, Tokyo, Japan). The membranes were reprobed with anti-GAPDH antibody, and analysed as above.

Immunofluorescence microscopy. Cells were cultured on cover glasses and fixed with $4.0 \%$ paraformaldehyde in phosphate buffered saline (PBS) for $20 \mathrm{~min}$ at $25^{\circ} \mathrm{C}$. The fixed cells were then washed with PBS for 5 min twice, and permeabilised with $0.1 \%$ Triton X-100 in PBS for $15 \mathrm{~min}$. The cells were blocked with $0.2 \%$ gelatin in PBS for $5 \mathrm{~min}$, and subsequently incubated with primary antibodies for $1 \mathrm{~h}$ at $4{ }^{\circ} \mathrm{C}$. The cells were washed with PBS-gelatin three times, and then incubated with the secondary antibody, Alexa Fluor 555-conjugated goat anti-mouse IgG. Nuclear staining was then performed using DAPI. The samples were subjected to microscopy using a laser-scanning confocal imaging system (LSM800; Carl Zeiss, AG, Jena, Germany) and analysed by Airyscan processing (ZEN2.3 software, Carl Zeiss). Subcellular distribution and colocalisation were quantified using Pearson's correlation coefficients. Quantitative data are presented as mean \pm standard deviation (SD). Statistical analyses were performed using Prism 7 (GraphPad, San Diego, CA, USA). Unpaired $t$-tests were used to identify differences when a significant difference $\left({ }^{\star} P<0.05\right.$, ${ }^{* *} P<0.01,{ }^{* *} P<0.001$, or $\left.{ }^{* * *} P<0.0001\right)$ was determined by analysis of variance.

Live cell imaging. HeLa cells expressing the GFP-Rab44 constructs were grown on glass-bottom dish, and fluorescently labelled with $0.1 \mu \mathrm{M}$ LysoTracker Red DND-99 (ThermoFisher) and $5 \mu \mathrm{g} / \mathrm{mL}$ Hoechst 33342 (Dojin) at $37^{\circ} \mathrm{C}$ for $30 \mathrm{~min}$. The cells were washed with media and observed under confocal microscopy. The area of LysoTracker-positive vesicles was calculated by employing ImageJ and subjected to statistical analysis.

Ionomycin, thapsigargin, and ML-SA1 treatment. HeLa cells grown on cover glasses were incubated with $2 \mu \mathrm{M}$ ionomycin, $10 \mu \mathrm{M}$ thapsigargin, or $10 \mu \mathrm{M} \mathrm{ML}-\mathrm{SA} 1$, for $15 \mathrm{~min}$ at $37^{\circ} \mathrm{C}$ in a $5 \% \mathrm{CO}_{2}$ atmosphere, and then immediately fixed with paraformaldehyde followed by immunofluorescence microscopy.

Received: 19 February 2020; Accepted: 15 October 2020

Published online: 05 November 2020

\section{References}

1. Wang, T., Li, L. \& Hong, W. SNARE proteins in membrane trafficking. Traffic 18, 767-775. https://doi.org/10.1111/tra.12524 (2017).

2. Gillingham, A. K. \& Munro, S. Transport carrier tethering-How vesicles are captured by organelles. Curr. Opin. Cell Biol. 59, 140-146. https://doi.org/10.1016/j.ceb.2019.04.010 (2019).

3. Stenmark, H. Rab GTPases as coordinators of vesicle traffic. Nat. Rev. Mol. Cell Biol. 10, 513-525. https://doi.org/10.1038/nrm27 28 (2009).

4. Hutagalung, A. H. \& Novick, P. J. Role of Rab GTPases in membrane traffic and cell physiology. Physiol. Rev. 91, 119-149. https:// doi.org/10.1152/physrev.00059.2009 (2011).

5. Zhen, Y. \& Stenmark, H. Cellular functions of Rab GTPases at a glance. J. Cell Sci. 128, 3171-3176. https://doi.org/10.1242/jcs.16607 4 (2015).

6. Pfeffer, S. R. Rab GTPases: Master regulators that establish the secretory and endocytic pathways. Mol. Biol. Cell 28, 712-715. https ://doi.org/10.1091/mbc.E16-10-0737 (2017).

7. Novick, P. Regulation of membrane traffic by Rab GEF and GAP cascades. Small GTPases 7, 252-256. https://doi.org/10.1080/21541 248.2016.1213781 (2016).

8. Barr, F. \& Lambright, D. G. Rab GEFs and GAPs. Curr. Opin. Cell Biol. 22, 461-470. https://doi.org/10.1016/j.ceb.2010.04.007 (2010).

9. Pereira-Leal, J. B., Hume, A. N. \& Seabra, M. C. Prenylation of Rab GTPases: Molecular mechanisms and involvement in genetic disease. FEBS Lett. 498, 197-200. https://doi.org/10.1016/s0014-5793(01)02483-8 (2001).

10. Leung, K. F., Baron, R. \& Seabra, M. C. Thematic review series: Lipid posttranslational modifications. geranylgeranylation of Rab GTPases. J. Lipid Res. 47, 467-475, https://doi.org/10.1194/jlr.R500017-JLR200 (2006).

11. Diekmann, Y. et al. Thousands of rab GTPases for the cell biologist. PLoS Comput. Biol. 7, e1002217. https://doi.org/10.1371/journ al.pcbi.1002217 (2011).

12. Surkont, J., Diekmann, Y. \& Pereira-Leal, J. B. Rabifier2: An improved bioinformatic classifier of Rab GTPases. Bioinformatics (Oxford, England), https://doi.org/10.1093/bioinformatics/btw654 (2016).

13. Klopper, T. H., Kienle, N., Fasshauer, D. \& Munro, S. Untangling the evolution of Rab G proteins: Implications of a comprehensive genomic analysis. BMC Biol. 10, 71. https://doi.org/10.1186/1741-7007-10-71 (2012).

14. Srikanth, S., Woo, J. S. \& Gwack, Y. A large Rab GTPase family in a small GTPase world. Small GTPases, 1-6, https://doi. org/10.1080/21541248.2016.1192921 (2016).

15. Srikanth, S. et al. A large Rab GTPase encoded by CRACR2A is a component of subsynaptic vesicles that transmit T cell activation signals. Sci Signal 9, ra31, https://doi.org/10.1126/scisignal.aac9171 (2016).

16. Shintani, M. et al. Characterization of Rab45/RASEF containing EF-hand domain and a coiled-coil motif as a self-associating GTPase. Biochem. Biophys. Res. Commun. 357, 661-667. https://doi.org/10.1016/j.bbrc.2007.03.206 (2007).

17. Yamaguchi, Y. et al. Rab44, a novel large Rab GTPase, negatively regulates osteoclast differentiation by modulating intracellular calcium levels followed by NFATc1 activation. Cell Mol. Life Sci. 75, 33-48. https://doi.org/10.1007/s00018-017-2607-9 (2018).

18. Kadowaki, T. et al. The large GTPase Rab44 regulates granule exocytosis in mast cells and IgE-mediated anaphylaxis. Cell Mol. Immunol. https://doi.org/10.1038/s41423-020-0413-z (2020).

19. Tokuhisa, M. et al. Expression and localisation of Rab44 in immune-related cells change during cell differentiation and stimulation. Sci. Rep. 10, 10728. https://doi.org/10.1038/s41598-020-67638-7 (2020). 
20. Simpson, J. C. et al. A role for the small GTPase Rab21 in the early endocytic pathway. J. Cell Sci. 117, 6297-6311. https://doi. org/10.1242/jcs.01560 (2004).

21. Gomes, A. Q. et al. Membrane targeting of Rab GTPases is influenced by the prenylation motif. Mol. Biol. Cell 14, 1882-1899. https://doi.org/10.1091/mbc.e02-10-0639 (2003).

22. Gifford, J. L., Walsh, M. P. \& Vogel, H. J. Structures and metal-ion-binding properties of the Ca2+-binding helix-loop-helix EFhand motifs. Biochem. J. 405, 199-221. https://doi.org/10.1042/bj20070255 (2007).

23. Gillingham, A. K. \& Munro, S. Long coiled-coil proteins and membrane traffic. Biochem. Biophys. Acta. 1641, 71-85. https://doi. org/10.1016/s0167-4889(03)00088-0 (2003).

24. Gillingham, A. K. \& Munro, S. Finding the golgi: Golgin coiled-coil proteins show the way. Trends Cell Biol. 26, 399-408. https:// doi.org/10.1016/j.tcb.2016.02.005 (2016).

25. Baba, K. et al. Different localization of lysosomal-associated membrane protein 1 (LAMP1) in mammalian cultured cell lines. Histochem. Cell Biol. 153, 199-213. https://doi.org/10.1007/s00418-019-01842-z (2020).

26. Pierzyńska-Mach, A., Janowski, P. A. \& Dobrucki, J. W. Evaluation of acridine orange, LysoTracker Red, and quinacrine as fluorescent probes for long-term tracking of acidic vesicles. Cytometry Part A 85, 729-737, https://doi.org/10.1002/cyto.a.22495 (2014).

27. DeVorkin, L. \& Gorski, S. M. LysoTracker staining to aid in monitoring autophagy in Drosophila. Cold Spring Harbor protocols 951-958, 2014. https://doi.org/10.1101/pdb.prot080325 (2014).

28. Kadowaki, T. et al. A two-component system regulates gene expression of the type IX secretion component proteins via an ECF sigma factor. Sci. Rep. 6, 23288. https://doi.org/10.1038/srep23288 (2016).

29. Takii, R., Kadowaki, T., Tsukuba, T. \& Yamamoto, K. Inhibition of gingipains prevents Porphyromonas gingivalis-induced preterm birth and fetal death in pregnant mice. Eur. J. Pharmacol. 824, 48-56. https://doi.org/10.1016/j.ejphar.2018.01.028 (2018).

30. Narahara, S. et al. KBTBD11, a novel BTB-Kelch protein, is a negative regulator of osteoclastogenesis through controlling Cullin3mediated ubiquitination of NFATc1. Sci. Rep. 9, 3523. https://doi.org/10.1038/s41598-019-40240-2 (2019).

\section{Acknowledgements}

This work was supported by JSPS KAKENHI grant numbers $17 \mathrm{H} 04379$ and 18K09536.

\section{Author contributions}

T.T. conceived the research. K.O. and T.K. designed and conducted the experiments. M.T. and Y.Y. contributed to plasmid construction. M.U. and T.T. analysed the data. T.K. and T.T. wrote the manuscript with the contributions from all authors. All authors approved the final manuscript.

\section{Competing interests}

The authors declare no competing interests.

\section{Additional information}

Supplementary information is available for this paper at https://doi.org/10.1038/s41598-020-75897-7.

Correspondence and requests for materials should be addressed to T.K. or T.T.

Reprints and permissions information is available at www.nature.com/reprints.

Publisher's note Springer Nature remains neutral with regard to jurisdictional claims in published maps and institutional affiliations.

(c) Open Access This article is licensed under a Creative Commons Attribution 4.0 International License, which permits use, sharing, adaptation, distribution and reproduction in any medium or format, as long as you give appropriate credit to the original author(s) and the source, provide a link to the Creative Commons licence, and indicate if changes were made. The images or other third party material in this article are included in the article's Creative Commons licence, unless indicated otherwise in a credit line to the material. If material is not included in the article's Creative Commons licence and your intended use is not permitted by statutory regulation or exceeds the permitted use, you will need to obtain permission directly from the copyright holder. To view a copy of this licence, visit http://creativecommons.org/licenses/by/4.0/.

(c) The Author(s) 2020 